\title{
Generalized Multiplexed Waveform Design Framework for Cost-Optimized MIMO Radar
}

\author{
Christian Hammes $\left.{ }^{(}\right)$, Member, IEEE, Bhavani Shankar M. R. (), Senior Member, IEEE, \\ and Björn Ottersten ${ }^{(0)}$, Fellow, IEEE
}

\begin{abstract}
Cost-optimization through the minimization of hardware and processing costs with minimal loss in performance is an interesting design paradigm in evolving and emerging MultipleInput-Multiple-Output (MIMO) radar systems. This optimization is a challenging task due to the increasing Radio Frequency (RF) hardware complexity as well as the signal design algorithm complexity in applications requiring high angular resolution. Towards addressing these, the paper proposes a low-complexity signal design framework, which incorporates a generalized time multiplex scheme for reducing the RF hardware complexity with a subsequent discrete phase modulation. The scheme further aims at achieving simultaneous transmit beamforming and maximum virtual MIMO aperture to enable better target detection and discrimination performance. Furthermore, the paper proposes a low-complexity signal design scheme for beampattern matching in the aforementioned setting. The conducted performance evaluation indicates that the listed design objectives are met.
\end{abstract}

Index Terms-Angle-Doppler coupling suppression, blockcirculant decomposition, MIMO radar, multiplexing, noniterative algorithm, transmit beamforming, virtual MIMO, convex optimization, finite alphabets, multiple phase shift keying.

\section{INTRODUCTION}

$\mathbf{H}$ IGH angular resolution is particularly interesting for imaging and classification applications. However, compared to LIDAR or camera based systems, radar technology suffers from achieving high angular resolution mainly due to physical limitations on the antenna aperture size relative to the signal carrier wavelength [1]. In an attempt to overcome the trade-off between angular resolution and aperture size, the large number of proposed approaches can be categorized majorly into: Synthetic Aperture Radar (SAR) and MultipleInput-Multiple-Output (MIMO) radar. While SAR approaches exploit the physical motion of the radar platform to span up a synthetic antenna array [2], MIMO approaches exploit the diversity offered by different antenna elements [3], [4]. Since SAR approaches work well only when the radar platform trajectory is

Manuscript received April 20, 2020; revised September 28, 2020; accepted November 10, 2020. Date of publication November 27, 2020; date of current version December 23, 2020. The work of Christian Hammes was supported by FNR Luxembourg, Industrial Fellowship Project DYNAMO. The work of Bhavani Shankar M. R. and Björn Ottersten were supported in part by ERC Advanced Grant AGNOSTIC (ID: 742648). The associate editor coordinating the review of this manuscript and approving it for publication was Dr. Fabiola Colone. (Corresponding author: Bhavani Shankar M. R.)

The authors are with the Interdisciplinary Centre for Security, Reliability and Trust, University of Luxembourg, 1855 Luxemburg, Luxembourg (e-mail: christian.hammes@uni.lu; Bhavani.Shankar@uni.lu; bjorn.ottersten@uni.lu).

Digital Object Identifier 10.1109/TSP.2020.3040859 precisely measured using another sensor [2], we focus on MIMO approaches, where imaging and classification can be performed with a standalone radar system.

Scaling the aperture size can be practically impossible due to form factor and/ or cost restrictions on the underlying application. On the other hand, given a form factor, an interesting practical approach is to minimize the cost relative to the array length with minimum performance loss. In this context, the key cost categories and the corresponding determining factors include, (i) the hardware cost largely determined by the number of Radio Frequency (RF) channels, and (ii) the processing cost largely determined by the computational resources needed for each processing interval in order to achieve real time capability [5]. This paper focuses on transmit processing to achieve high angular resolution while addressing the aforementioned cost factors. To position the contributions of the paper in this context, it becomes essential to quickly summarize the implications of such a cost optimization.

Minimizing RF Units: A transmit RF unit is able to modulate the signal in frequency, phase or amplitude independently before transmission. Higher number of independent waveforms/ phase modulations naturally increases the number of RF units. An implication of this is the consideration of time-multiplexed virtual MIMO solutions for utilizing lower number of RF units. Virtual MIMO array is a well known approach for reducing the number of RF channels, and, hence the hardware complexity in MIMO radar applications, while offering higher angular resolution due to waveform diversity [4], [6]-[11]. Assuming one channel per antenna element, the number of RF channels relative to the aperture size is reduced, but typically at the expense of designing orthogonal transmit signals [7]-[11]. A further simultaneous reduction of the number of RF channels and computational complexity of waveform design can be obtained by using Time Division Multiplex (TDM) modulation [12]. While TDM MIMO is effective and is of low cost, beamforming is inherently impossible due to the single antenna excitation. This reduces the receiver Signal to Noise Ratio (SNR) impacting performance for weak targets. Finally, the number of independent receive RF units can be characterized by the number of unique analogue to digital converters.

Another cost reducing aspect is the efficient resource utilization of the underlying hardware. Power efficient design enables better signal quality at the receiver; in this context, the best power efficiency is offered by imposing constant modulus property on the transmitted signal [11]. Motivated by this, several works 


\begin{tabular}{|l|l|l|l|l|}
\hline State-of-the-Art & $\begin{array}{l}\text { Angle- } \\
\text { Doppler } \\
\text { Coupling }\end{array}$ & $\begin{array}{l}\text { Virtual } \\
\text { MIMO }\end{array}$ & $\begin{array}{l}\text { Beam- } \\
\text { forming } \\
\text { Capability }\end{array}$ & $\begin{array}{l}\text { Time } \\
\text { Multiplex }\end{array}$ \\
\hline Category 1 & Yes & Yes & No & TDM \\
\hline Category 2 & No & No & Yes & general \\
\hline Category 3 & No & Yes & Yes & No \\
\hline Category 4 & No & Yes & No & No \\
\hline Category 5 & No & No & Yes & No \\
\hline This paper & Yes & Yes & Yes & general \\
\hline
\end{tabular}

Fig. 1. State-of-the-Art vs. our contribution. Category 1: [12], [18]; Category 2: [19]-[21]; Category 3: [22], [23]; Category 4:[7]-[11]; Category 5:[13]-[15], [17], [24]-[27], [27]-[35], [36].

consider using constant modulus, unimodular and related peak to average ratio (PAR) as constraints for various applications including ambiguity function design [9], [13]-[16] . However, the use of constant modulus signals for time-multiplexed architectures imposes a non-convex constraint on the waveform structure and complicates the design procedure. Naturally, the design becomes challenging when only minimal computational resources are available.

Minimizing Computational Resources: These resources are determined by the complexity of signal design and of receive processing algorithms. This implies infeasibility of implementing elaborate optimization methodologies to ensure real-time operations in dynamic scenarios at low cost. This motivates lowering the dimensions/ complexity of the optimization problem. This reduction can be achieved by striving for closed form expressions instead of summations [17] and by reducing the number of optimization variables.

Fig. 1 illustrates the canvas of relevant literature towards highlighting the contributions of this paper in the context of cost optimization. Fig. 1 indicates that the prior-art addresses only a selection of issues, but none consider a holistic approach towards cost minimization. In particular, following aspects are not addressed simultaneously.

- Generalized Multiplexing: The number of RF units (subsequently referred to as number of channels $N_{c}$ ) being between one and the number of transmit antennas $N_{T}$,

- Receive and transmit array configuration satisfying the virtual MIMO concept,

- Investigating Angle-Doppler coupling as a consequence of multiplexing and slow-time modulation,

- Beamforming capability for an enhanced Signal to Noise Ratio and therefore better target detection performance.

While considering the aforementioned aspects offers a holistic cost reduction approach with additional flexibility, the design is challenging and more complicated than a mere aggregation of individual aspects. The paper addresses this important gap through a novel waveform design to meet the following two major objectives: (1) General Multiplex Framework enabling beamforming, (2) Low complexity simultaneous Beamforming and virtual MIMO paradigm with discrete-phase signals.

Towards meeting the objectives, the paper considers colocated transmit/ receive antennas yielding a virtual array with maximum length and intends to achieve maximum angular resolution for the entire virtual array. Further, the key contributions to meet the objectives and scale MIMO radar arrays from a cost-optimization perspective include:

- General multiplex framework enabling beamforming $(1<$ $N_{c}<N_{T}$ ): In order to overcome the lack of beamforming in TDM modulation using a limited number of RF units, several works report on a generalized TDM approach, where the number of RF channels is between one and the number of transmit antennas [19]-[21]. Unlike these works, our paper considers both the virtual MIMO advantages and unimodular designs. A specific multiplexing design with two channels and QPSK modulation was considered in [37]. This work builds on [37] and provides a systematic approach to signal design when generalizing the number of antennas excited and the modulation alphabet. In fact, the design in [37] is a special case of the current work . This generalization enables beamforming capabilities with a lower number of RF units and offers flexibility to the system designer.

- Discrete phase slow-time modulation: We consider a lowcomplexity, but not necessarily limiting, slow-time modulated continuous wave $(\mathrm{CW})$ transmissions, i. e, a $\mathrm{CW}$ pulse modulated on an inter-chirp basis. We introduce a discrete phase constraint (discretized case of the unimodular design constraint) on this slow-time modulation to enhance power efficiency in a general multiplex setting. Following [22], [31], we consider a uniform elemental power constraint on the transmit array.

- Reduced complexity waveform design framework supporting beamforming in virtual MIMO paradigm: An optimization problem for beampattern design based on the aforementioned constraints is derived for the considered virtual MIMO. Exploiting the structure in the problem formulation, a simplification based on the Block Circulant Decomposition (BCD) is obtained. The novel $\mathrm{BCD}$ approach has few variables, implicitly accounts for the uniform power constraint, inherently satisfies good crosscorrelation properties of the transmitted signals and offers maximal virtual antenna aperture. This leads to a design framework with a lower dimensional problem space.

Leveraging the structured BCD paradigm, the well-known dictionary based approach is adapted to provide an elegant solution for the BCD relaxed problem. The dictionary contains the array excitation values as atoms and the novelty lies is relating the beampattern optimization problem to these. Further the generation of dictionary including general multiplex and discrete phase modulation design constraint is novel; in special cases, structured dictionary form exists leading to closed-form expression for the signal. Unlike the rigorous and complicated optimization methodologies like [9], the dictionary based approach allows for a fast and efficient implementation of the optimization. This approach, hitherto not considered, enables easier adaptation of transmit waveforms. Further, the closed-form signal design in [23] is a special case of the current work for a specific setting of modulation alphabet and RF channels used. 
- Mitigation of Angle-Doppler Coupling: In addition to the cost parameters, we also investigate the angle-Doppler coupling issue - a well known problem in TDM MIMO application for a slow-time modulation scheme [12], [18]. The resulting coupling is investigated and a random column permutation of the signal matrix is analysed for its mitigation. This mitigation is not considered in the related works [23], [37].

Towards presenting the contributions, in Section II, the signal model for a slow-time modulated $\mathrm{CW}$ signal is derived for a co-located virtual MIMO structure with a matched filter receive processing scheme. Section III formulates an optimization problem for the slow-time modulation signal design. It is driven by the cost-optimization outlook, imposing constraints on number of transmit RF units, design complexity and operational efficiency. The optimization problem is hard to solve and therefore a low complexity dictionary based optimization framework is proposed in Section IV. Section V investigates the adaptation of the design to mitigate angle-Doppler coupling and the results in Section VI corroborate the design claims. Section VII draws key conclusions.

In this work, the operator $\|\cdot\|_{p}$ defines the $l_{p}$-norm. A matrix entry is defined by $[\cdot]_{\eta, \zeta}$, where $\eta$ denotes the row index and $\zeta$ the column index. A vector entry $[\cdot]_{\eta}$ is defined by one index $\eta$. The $E\{\cdot\}$ denotes the expectation operator. The set of complex numbers is defined as $\mathbb{C}$, while $j=\sqrt{-1}$ represents the complex number. The Kronecker and the Hadamard product is defined as $\otimes$ and $\circ$, respectively. Further, ${ }^{T},{ }^{H}$ denote the transpose and Hermitian operations,

\section{SYSTEM MODEL}

The underlying radar system comprises a local oscillator, generating a train of $\mathcal{I}_{c}$ consecutive pulses in each Coherent Processing Interval (CPI). This pulse train has a center frequency $f_{0}$ and a modulation bandwidth $B$. Each pulse of duration $T_{c}$ is further modulated in the transmit modulation unit on a pulse-by-pulse manner. Further, the transmit modulation unit comprises $N_{c}$ phase modulation channels; each channel has the capability to modulate the pulse using $N_{p}$ distinct phase stages that are distributed uniformly around the unit circle. The $N_{c}$ phase modulated channels are multiplexed among $N_{T}$ transmit antennas, where $N_{c} \leq N_{T}$. This is undertaken to reflect the capabilities of the emerging hardware systems.

The $N_{R}$ receive antennas are arranged in a Uniform Linear Array (ULA) configuration along $x$-axis with an inter-element spacing of $d_{R}=\frac{\lambda}{2}$. The transmit antennas are also uniformly mounted along the $x$-axis with an inter-element spacing of $d_{T}=N_{R} \frac{\lambda}{2}$. This leads to a sparse transmit ULA. The transmitted signal propagates towards $K$ targets assumed to be located in the plane-wave far-field region. The back-scattered signal is captured by $N_{R}$ receive antennas, each having its own digital processing chain leading to the use of $N_{R}$ Analog to Digital Converters (ADCs). The $N_{R}$ received signals are then processed by a bank of matched filters in the digital domain. The information gathered at the output of the matched filter will be used later in the proposed signal design.

\section{A. Transmitted Signal}

Since the receiver operates in the digital domain, the signal model of the transmitted waveform is also described in that domain. Each pulse consists of $\mathcal{I}_{s}$ samples with $i_{s}$ denoting the sample index. The pulse vector, $\mathbf{p} \in \mathbb{C}^{\mathcal{I}_{s} \times 1}$, represents the sampled version of a pulse $p(t)$, i.e., $[\mathbf{p}]_{i_{s}}=p\left(i_{s} T_{s}\right)$, where $T_{s}$ denotes the sampling time. As indicated earlier, the transmitted waveform is a train of modulated pulses. To effect this inter-pulse modulation, the transmitted signal during the $i_{c}$-th pulse radiated from the $n$-th transmit antenna takes the form, $s_{i_{c}}(n) \mathbf{p}$, where $s_{i_{c}}(n)$ corresponds to the inter-pulse modulation symbol. Further, $s_{i_{c}}(n) \in \Omega_{0}$, where the set $\Omega_{0}=$ $\{0\} \cup \Omega$ includes zero to model the multiplexing, and the set $\Omega=\left\{1, \exp \left(j \frac{2 \pi}{N_{p}}\right) \cdots \exp \left(j \frac{2 \pi\left(N_{p}-1\right)}{N_{p}}\right)\right\}$ comprises $N_{p}$ distinct uniformly distributed phase stages. This choice of the modulation symbols enables low-complexity realizable radars.

Let $\mathbf{s}_{i_{c}} \in \Omega_{0}^{N_{T} \times 1}$ denote the stacking of the $N_{T}$ transmit antenna weightings, $\left\{s_{i_{c}}(n)\right\}_{n=1}^{N_{T}}$, for the $i_{c}$-th pulse. The modulation vector, $\mathbf{s} \in \Omega_{0}^{\mathcal{I}_{c} N_{T} \times 1}$, further contains the antenna weighting vectors $\left\{\mathbf{s}_{i_{c}}\right\}_{i_{c}=1}^{\mathcal{I}_{c}}$ across the $\mathcal{I}_{c}$ pulses,

$$
\mathbf{s}=\left(\mathbf{s}_{1}^{T} \cdots \mathbf{s}_{i_{c}}^{T} \cdots \mathbf{s}_{\mathcal{I}_{c}}^{T}\right)^{T} .
$$

The transmit signal vector samples $\mathbf{x} \in \mathbb{C}^{\mathcal{I}_{s} \mathcal{I}_{c} N_{T} \times 1}$ across $N_{T}$ antennas and $\mathcal{I}_{c}$ pulses can be denoted as the Kronecker product of inter-pulse signal modulation vector, $\mathbf{s}$ and the sampled pulse vector,

$$
\mathbf{x}=\mathbf{s} \otimes \mathbf{p} .
$$

Signal design involves both $\mathbf{p}$ and $\mathbf{s}$. However, the design of $\mathbf{p}$ has been extensively considered in prior-art and the focus of this paper will be on the design of $\mathbf{s}$ for enhanced angle-Doppler resolution.

\section{B. System Transfer Function and Received Signal}

It is reasonable to assume that the pulse duration is much longer than the propagation delay for the target in far-field, i.e., $T_{c} \gg \frac{r_{\max }}{c_{0}}$, where $c_{0}$ is the speed of light and $r_{\max }$ is the maximum target range. Further, if the maximum target velocity is small such that its position can be considered as being constant during the pulse duration $T_{c}$, the Doppler and range information become separable. In fact, the range and Doppler information can be obtained from the fast-time (within a pulse), and slow-time domains (across pulses) respectively.

The radar environment comprises $K$ targets whose Radar Cross Sections (RCS) follow the Swerling-I statistics [38]. Particularly, the RCS for $\kappa$-th target, denoted by $\alpha_{\kappa} \in \mathbb{C}$, is a statistical parameter and the RCS of distinct targets are independent of each other. The angular information for the $\kappa$-th target is modelled into the receive steering vector $\mathbf{a}_{R, \kappa} \in \mathbb{C}^{N_{R} \times 1}$ and the transmit steering vector $\mathbf{a}_{T, \kappa} \in \mathbb{C}^{N_{T} \times 1}$, as

$$
\begin{aligned}
{\left[\mathbf{a}_{R, \kappa}\right]_{m} } & =\exp \left(j k_{0} \sin \left(\phi_{\kappa}\right) d_{R}(m-1)\right) \\
{\left[\mathbf{a}_{T, \kappa}\right]_{n} } & =\exp \left(j k_{0} \sin \left(\phi_{\kappa}\right) d_{T}(n-1)\right),
\end{aligned}
$$

with $k_{0}=\frac{2 \pi}{\lambda}$ (free-space wavenumber) and $\phi_{\kappa}$ being the angle of arrival of the $\kappa$-th target. The received signal experiences a 
Doppler shift $\omega_{\kappa}$ for the $\kappa$-th target and its effect is modelled using the diagonal Doppler matrix $\mathbf{D}_{\omega_{k}} \in \mathbb{C}^{\mathcal{I}_{c} \times \mathcal{I}_{c}}$,

$$
\mathbf{D}_{\omega_{\kappa}}=\operatorname{diag}\left(\exp \left(j \omega_{\kappa} T_{c}\right), \ldots, \exp \left(j \omega_{\kappa} T_{c} \mathcal{I}_{c}\right)\right) .
$$

On the other hand, the range information for the $\kappa$-th target appears as a time shift of the pulse vector $\mathbf{p}$. The time shift and the $\kappa$-th target range information are modelled by a shift matrix $\mathbf{J}_{\kappa} \in\{0 ; 1\}^{\mathcal{I}_{s} \times \mathcal{I}_{s}}[39]$. The received signal vector corresponding to the $i_{c}$ th pulse at the $r$ th receive antenna, denoted by $\mathbf{z}_{r, i_{c}} \in$ $\mathbb{C}^{\mathcal{I}_{s} \times 1}$, then takes the form,

$$
\mathbf{z}_{r, i_{c}}=\sum_{\kappa=1}^{K} \alpha_{\kappa}\left[\mathbf{a}_{R, \kappa}\right]_{r} \sum_{m=1}^{N_{T}}\left[\mathbf{a}_{T, \kappa}\right]_{m} s_{i_{c}}(m)\left[\mathbf{D}_{\omega_{\kappa}}\right]_{i_{c}, i_{c}} \mathbf{J}_{\kappa} \mathbf{p} .
$$

Let $\mathbf{z} \in \mathbb{C}^{N_{R} \mathcal{I}_{c} \mathcal{I}_{s} \times 1}$ denote the received signal vector obtained by stacking $\mathbf{z}_{r, i_{c}}$ of (6) over the $N_{R}$ received antennas and $\mathcal{I}_{c}$ pulses. To model $\mathbf{z}$, it is convenient to exploit the inter-pulse modulation and define the inter-pulse transfer function $\mathbf{H}_{\kappa} \in \mathbb{C}^{\mathcal{I}_{c} N_{R} \times \mathcal{I}_{c} N_{T}}$ for the $\kappa$-th target as [37],

$$
\mathbf{H}_{\kappa}=\alpha_{\kappa}\left(\mathbf{a}_{R, \kappa} \mathbf{a}_{T, \kappa}^{H}\right) \otimes \mathbf{D}_{\omega_{\kappa}} .
$$

Using (6) and (7), it follows that,

$$
\mathbf{z}=\sum_{\kappa=1}^{K}\left(\mathbf{H}_{\kappa} \mathbf{s}\right) \otimes\left(\mathbf{J}_{\kappa} \mathbf{p}\right)=\left(\sum_{\kappa=1}^{K} \mathbf{H}_{\kappa} \otimes \mathbf{J}_{\kappa}\right)(\mathbf{s} \otimes \mathbf{p}) .
$$

It follows from (8) that the slow-time information, $\mathbf{H}_{\kappa} \mathbf{s}$, is separable from the fast-time information $\mathbf{J}_{\kappa} \mathbf{p}$ due to the Kronecker structure. Further, since this paper focusses on inter-pulse modulation, it suffices to only investigate the slow-time information. Referring to (8), the term of interest is the slow-time receive signal vector $\mathbf{y} \in \mathbb{C}^{N_{R} \mathcal{I}_{c} \times 1}$ defined as,

$$
\mathbf{y}=\sum_{\kappa=1}^{K} \mathbf{H}_{\kappa} \mathbf{s}
$$

Due to the definition of $\mathbf{H}_{\kappa}$ in (7), the signal design only affects the angle-Doppler response of each target. In the sequel, only the inter-pulse receive vector in (9) is considered.

\section{Matched Filter (MF)}

The information in the received signal is extracted by a matched filter (MF) with the coefficients $\mathbf{y}_{M}=\mathbf{H}_{M}$ s. Since the RCS is not known a priori, the MF is parametrized by $\omega_{M}$ and $\phi_{M}$ similar to the receive signal model as,

$$
\mathbf{H}_{M}=\left(\mathbf{a}_{R, M} \mathbf{a}_{T, M}^{H}\right) \otimes \mathbf{D}_{M},
$$

where $\mathbf{a}_{T, M}, \mathbf{a}_{R, M}, \mathbf{D}_{M}$ are obtained from (3), (4), (5) by using design parameters $\left\{\phi_{M}, \omega_{M}\right\}$ instead of $\left\{\phi_{\kappa}, \omega_{\kappa}\right\}$ respectively. Since the target RCS follow a Swerling-I model [38], the parameter of interest is the ensemble average of the squared matched filter output over the random $\left\{\alpha_{\kappa}\right\}$. Denoting $\mu$ as the average matched filter output, it follows that,

$$
\mu=E\left\{\left|\mathbf{y}_{M}^{H} \mathbf{y}\right|^{2}\right\}=E\left\{\left|\sum_{\kappa=1}^{K} \mathbf{s}^{H} \mathbf{H}_{M}^{H} \mathbf{H}_{\kappa} \mathbf{s}\right|^{2}\right\} .
$$

Exploiting the block diagonal structure of $\mathbf{H}_{M}$ and $\mathbf{H}_{\kappa}$, (kindly refer to (7), (10), respectively), the expression $\sum_{\kappa=1}^{K} \mathbf{s}^{H} \mathbf{H}_{M}^{H} \mathbf{H}_{\kappa}$ s above can written as a sum over $\mathcal{I}_{c}$ blocks. Further, the uncorrelated RCS fluctuations of distinct targets result in the expected matched filter output being a superposition of $K$ target responses,

$$
\begin{aligned}
& \mu=\sum_{\kappa=1}^{K} \sigma_{\kappa}^{2}\left|\mathbf{a}_{R, M}^{H} \mathbf{a}_{R, \kappa}\right|^{2} \\
& \times\left|\mathbf{a}_{T, M}^{H}\left(\sum_{i_{c}=1}^{\mathcal{I}_{c}} \mathbf{s}_{i_{c}} \mathbf{s}_{i_{c}}^{H} \exp \left(-j\left(\omega_{M}-\omega_{\kappa}\right) T_{c} i_{c}\right)\right) \mathbf{a}_{T, \kappa}\right|^{2},
\end{aligned}
$$

where $\sigma_{\kappa}^{2}=E\left\{\left|\alpha_{k}\right|^{2}\right\}$ denotes the variance of the $\kappa$-th attenuation factor and recall that $\mathbf{s}_{i_{c}} \in \Omega_{0}^{N_{T} \times 1}$ is the modulation vector for the $i_{c}$-th pulse.

The Doppler shift is omitted explicitly in the initial investigation to ease waveform design. The waveforms thus designed will then be adapted in Section V to cater to Doppler. Thus, without considering the Doppler shift, (12) reduces to $\mu=\sum_{\kappa=1}^{K} \sigma_{\kappa}^{2}\left|\mathbf{a}_{R, M}^{H} \mathbf{a}_{R, \kappa}\right|^{2}\left|\mathbf{a}_{T, M}^{H}\left(\sum_{i_{c}=1}^{\mathcal{I}_{c}} \mathbf{s}_{i_{c}} \mathbf{s}_{i_{c}}^{H}\right) \mathbf{a}_{T, \kappa}\right|^{2}$. Recalling that $\mathbf{a}_{T, M}$ and $\mathbf{a}_{T, \kappa}$ are functions of the corresponding angles of arrival $\phi_{M}$ and $\phi_{k}$ respectively, the term $\mathbf{a}_{T, M}^{H}\left(\sum_{i_{c}=1}^{\mathcal{I}_{c}} \mathbf{s}_{i_{c}} \mathbf{s}_{i_{c}}^{H}\right) \mathbf{a}_{T, \kappa}$ is a function of $\phi_{M}$ and $\phi_{\kappa}$. To highlight this dependence, several works in literature [15], [22], [33], [37] define the cross-correlation beampattern as,

$$
P\left(\phi_{\kappa}, \phi_{M}\right)=\mathbf{a}_{T, M}^{H}\left(\sum_{i_{c}=1}^{\mathcal{I}_{c}} \mathbf{s}_{i_{c}} \mathbf{s}_{i_{c}}^{H}\right) \mathbf{a}_{T, \kappa} .
$$

In fact, when the actual target angle coincides with the MF design, $P\left(\phi_{\kappa}, \phi_{M}\right)$, reduces to the radiated beampattern along $\phi_{M}$. For other settings, $P\left(\phi_{\kappa}, \phi_{M}\right)$ illustrates the resolution capability of the MF output. This capability to encompass different notions highlights the utility of $P\left(\phi_{\kappa}, \phi_{M}\right)$ in waveform design and will be now be explored further.

\section{Signal Design Objective}

Let the signal matrix $\mathbf{S} \in \Omega_{0}^{N_{T} \times \mathcal{I}_{c}}$ contain all the modulation vectors $\mathbf{s}_{i_{c}}$ in its columns. Further, let $\mathbf{R}_{s} \in \mathbb{C}^{N_{T} \times N_{T}}$ be the covariance matrix of $\mathbf{S}$. These take the form,

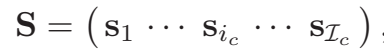

$$
\begin{aligned}
& \mathbf{R}_{s}=\sum_{i_{c}=1}^{\mathcal{I}_{c}} \mathbf{s}_{i_{c}} \mathbf{s}_{i_{c}}^{H}=\mathbf{S} \mathbf{S}^{H},
\end{aligned}
$$

From (13), it is clear. that $\mathbf{R}_{s}$ is central to the design of the auto- and cross-correlation beampatterns. In fact, for orthogonal transmit signals, $\mathbf{R}_{s}$ is diagonal and the virtual MIMO concept is then satisfied resulting in the maximum angular resolution [6]. However, for orthogonal signals, the transmit radiation pattern is inherently isotropic and results in a SNR loss [3]. On the other hand, beamforming with coherent transmission improves SNR, but has a poor angular resolution. 
In view of the discussion above, the objective is to design a transmit signal such that a desired radiation pattern is achieved, while also enabling the virtual MIMO paradigm for maximum angular resolution. Towards this, the design of the signal dependent cross-correlation beampattern, $P\left(\phi_{\kappa}, \phi_{M}\right)$ is investigated. It is well known that orthogonal transmit signals achieve best resolution performance for the virtual MIMO configuration [6] and $P\left(\phi_{\kappa}, \phi_{M}\right)$ also reflects this property. Further, the crosscorrelation beam $P\left(\phi_{\kappa}, \phi_{M}\right)$ is shown to be related to the transmit signal covariance matrix $\mathbf{R}_{s}$ [22]. Therefore, the crosscorrelation beampattern incorporates the desired properties and also provides a suitable design objective for enhanced angular resolution and beampattern design.

\section{BEAMPATTERN FORMULATION BASED ON BLOCK Circulant Signal DESIGN}

Based on the derived model, the inter-pulse modulation matrix $\mathbf{S}$ chosen from $\Omega_{0}$ needs to be designed such that a desired cross-correlation beampattern is achieved. Towards this, the cross-correlation beampattern is investigated in more detail and a suitable signal design framework is formulated.

\section{A. Cross-Correlation Beampattern and Signal Covariance}

This section provides a formulation for the design of the signal covariance matrix to achieve a desired beam-pattern. The first step in this direction is the sampling of $P(\cdot)$ in (13) and the same is described below.

1) Sampled Cross-Correlation Beampattern: Let the 2-D desired cross-correlation beampattern, $P\left(\phi_{\kappa}, \phi_{M}\right)$, be sampled along $\phi_{\kappa}$ and $\phi_{M}$ with $N_{\kappa}$ and $N_{M}$ samples respectively. These samples can be compactly arranged in a matrix, $\overline{\mathbf{P}} \in \mathbb{C}^{N_{M} \times N_{\kappa}}$ with $[\overline{\mathbf{P}}]_{p, q}$ being $P(\cdot, \cdot)$ evaluated at the $(p, q)$ th sample of $\phi_{M}$ and $\phi_{\kappa}$ respectively. Let $\mathbf{a}_{T, M_{p}}, \mathbf{a}_{T, \kappa_{q}}$ denote the transmit array and matched filter steering vectors obtained by using the $p, q$ sample of $\phi_{M}$ and $\phi_{\kappa}$ in (3) and (4) respectively. Then, letting $\overline{\mathbf{A}}_{T, \kappa}=\left(\mathbf{a}_{T, \kappa_{1}}, \ldots, \mathbf{a}_{T, \kappa_{N_{\kappa}}}\right) \in$ $\mathbb{C}^{N_{T} \times N_{\kappa}}$ and $\overline{\mathbf{A}}_{T, M}=\left(\mathbf{a}_{T, M_{1}}, \ldots, \mathbf{a}_{T, M_{N_{M}}}\right) \in \mathbb{C}^{N_{T} \times N_{M}}$, it follows from (13) that $\overline{\mathbf{P}}=\overline{\mathbf{A}}_{T, M}^{H} \mathbf{R}_{s} \overline{\mathbf{A}}_{T, \kappa}$. If the number of samples for the matched filter steering matrix, $\overline{\mathbf{A}}_{T, M}$ and the transmit array steering matrix, $\overline{\mathbf{A}}_{T, \kappa}$ are set identically to $N_{0}$, i.e., $N_{0}=N_{\kappa}=N_{M}$, then $\overline{\mathbf{A}}_{T}=\overline{\mathbf{A}}_{T, M}=\overline{\mathbf{A}}_{T, \kappa} \in \mathbf{C}^{N_{T} \times N_{0}}$ and the cross-correlation beampattern can be written as,

$$
\begin{aligned}
\overline{\mathbf{P}} & =\overline{\mathbf{A}}_{T}^{H} \mathbf{R}_{s} \overline{\mathbf{A}}_{T}, \\
{\left[\overline{\mathbf{A}}_{T}\right]_{n, l} } & =e^{j \frac{2 \pi}{\lambda} \sin \left(\phi_{l}\right)(n-1) d_{T}},
\end{aligned}
$$

where $\overline{\mathbf{A}}_{T}$ is the ULA steering matrix. It now remains to describe the samples $\left\{\phi_{k}\right\}$; in fact, the angular domain is sampled nonuniformly in $\phi_{l}$, such that,

$$
\sin \left(\phi_{l}\right)=2\left(l / N_{0}\right),-N_{0} / 2 \leq l \leq N_{0} / 2 .
$$

The non-uniform sampling is reasonable since the field of view is between $\pm \frac{\pi}{2}$ and the sine function is monotonic within this domain. Use of (18) and $d_{T}=N_{R} \frac{\lambda}{2}$ from Section II in (17), yields a steering vector matrix having a Discrete Fourier Transform (DFT) like structure,

$$
\left[\overline{\mathbf{A}}_{T}\right]_{n, l}=e^{j \frac{2 \pi N_{R}(n-1)(l-1)}{N_{0}}}, n \in\left[1, N_{T}\right], l \in\left[1, N_{0}\right] .
$$

The choice of $N_{0}$ should be reflective of the resolution limits/ target discrimination capabilities offered by the system. In this context, orthogonal signals are considered as they achieve the maximum possible angular resolution and, therefore, the best target discrimination [6]. The cross-correlation beampattern specialized for orthogonal transmit signals, denoted by $\mathbf{P}_{\perp}$, is defined as,

$$
\mathbf{P}_{\perp}=\gamma \overline{\mathbf{A}}_{T}^{H} \mathbf{I}_{N_{T}} \overline{\mathbf{A}}_{T},
$$

where transmit signal from each antenna is assumed to have the power $\gamma \geq 0$. The elements of $\mathbf{P}_{\perp}$ have the structure of a sinus-cardinal function,

$$
\begin{aligned}
& {\left[\mathbf{P}_{\perp}\right]_{n, k}=\gamma \sum_{l=1}^{N_{T}}\left[\overline{\mathbf{A}}_{T}^{H}\right]_{n, l}\left[\overline{\mathbf{A}}_{T}\right]_{l, k}} \\
& =\gamma \sum_{l=0}^{N_{T}-1} \exp \left(j \frac{4 \pi l d_{T}}{\lambda N_{0}}(k-n)\right), \\
& =\gamma \exp \left(j \frac{\left(N_{T}-1\right) 2 \pi d_{T}}{\lambda N_{0}}(k-n)\right) \frac{\sin \left(\frac{N_{T} 2 \pi d_{T}}{N_{0} \lambda}(k-n)\right)}{\sin \left(\frac{2 \pi d_{T}}{\lambda N_{0}}(k-n)\right)} .
\end{aligned}
$$

The structure of $\mathbf{P}_{\perp}$ shows the limit on physical resolution imposed by the antenna array size. When $N_{0}=N_{T} N_{R}$, the $N_{T} \times N_{R} N_{T}$ matrix $\overline{\mathbf{A}}_{T}$ in (19) reduces to,

$$
\begin{aligned}
\overline{\mathbf{A}}_{T} & =\mathbf{A}_{T} \otimes \mathbf{1}_{N_{R}}^{T}, \\
{\left[\mathbf{A}_{T}\right]_{n, l} } & =e^{j \frac{2 \pi(n-1)(l-1)}{N_{T}}}, n, l \in\left[1, N_{T}\right]
\end{aligned}
$$

where $\mathbf{A}_{T}$ is the $N_{T} \times N_{T}$ scaled DFT matrix and $\mathbf{1}_{N_{R}}$ is a column of $N_{R}$ ones. It can be shown that $\mathbf{P}_{\perp}=N_{T} \mathbf{I} \otimes \mathbf{1}_{N_{R}} \mathbf{1}_{N_{R}}^{T}$, which clearly exhibits a periodic structure. This is not surprising since the sparse transmit ULA inherently imposes a periodic structure on the beampattern and this periodicity is well captured by the choice of $N_{0}=N_{T} N_{R}$; its impact will be studied shortly.

Due to the structure of $\overline{\mathbf{A}}_{T}, N_{0}>N_{R} N_{T}$, does not provide additional information. Further, $N_{0}<N_{R} N_{T}$ does not capture the periodicity well. Hence, it suffices to consider $N_{0}=N_{T} N_{R}$. Furthermore, the radiation pattern $(k=n)$ for the orthogonal transmit signal case is always constant, leading to an isotropic radiation pattern discussed earlier.

2) Beampattern With Sparse Transmit Array: As noted earlier, the replication of $N_{R}$ scaled DFT matrices in (22) is justified by the sparse nature of the transmit ULA. This structure simplifies $\overline{\mathbf{P}}$ in (16), as $\overline{\mathbf{P}}=\left(\mathbf{A}_{T}^{H} \mathbf{R}_{s} \mathbf{A}_{T}\right) \otimes \mathbf{1}_{N_{R}} \mathbf{1}_{N_{R}}^{T}$, which, as in the earlier section, illustrated periodicity.

Due to the induced periodicity, it suffices to consider only one period of the transmit radiation pattern - the one that can actually be designed. This enables the reasonable simplification where only the principle $N_{T} \times N_{T}$ matrix of $\overline{\mathbf{P}}$ in (16) is investigated henceforth (alternatively only the first $N_{T}$ columns 
of $\overline{\mathbf{A}}_{T}$ are investigated). Noting that the first $N_{T}$ columns of $\overline{\mathbf{A}}_{T}$ yield $\mathbf{A}_{T}$ (refer to (22)) and letting $\mathbf{P}$ to be the relevant $N_{T} \times N_{T}$ sub-matrix of $\overline{\mathbf{P}}$, it follows that,

$$
\mathbf{P}=\mathbf{A}_{T}^{H} \mathbf{R}_{s} \mathbf{A}_{T} .
$$

Equations (23), (24) will be used in the sequel as beam-steering matrix and transmit radiation pattern respectively.

3) Desired Beampattern and Signal Design: In order to relate the insights of the orthogonal transmit signal investigation to an arbitrary beampattern design, the desired beam has to be defined. Since the maximum resolution is desired and that $\operatorname{rank}(\mathbf{P}) \leq N_{T}$, the desired cross-correlation matrix, defined as $\mathbf{P}_{d} \in \mathbb{R}^{N_{T} \times N_{T}}$, is considered to be diagonal. The diagonal structure of the desired cross-correlation matrix reflects the need to span up the maximum virtual aperture. It accounts for maximum angular resolution with ease of separating the signals from different antennas [4], [6]. Further, the diagonal elements contain the desired radiation pattern vector $\mathbf{p}_{d} \in \mathbb{R}_{+}^{N_{T} \times 1}$ comprising non-negative elements,

$$
\mathbf{P}_{d}=\operatorname{diag}\left(\mathbf{p}_{d}\right) .
$$

With the desired pattern defined, it now remains to formulate a signal design (or inter-pulse modulation matrix) criteria. In order to ensure that the objective function satisfies the generalized multiplexing paradigm, only $N_{c}$ entries of each $\mathbf{s}_{i_{c}}$ are allowed to be non-zero. This is ensured through the use of a zero norm constraint. In fact, the use of zero norm constraint differentiates this paper from other approaches as discussed in the introduction. Further, an uniform power transmission from all antennas is desired [22]; this is taken into account by the diagonal elements of $\mathbf{R}_{s}$. Therefore, the design optimization problem can be formulated as,

$$
\begin{gathered}
\min _{\mathbf{S} \in \Omega_{0}^{N_{T} \times \mathcal{I}_{c}}}\left\|\mathbf{P}_{d}-\mathbf{A}_{T}^{H} \mathbf{R}_{s} \mathbf{A}_{T}\right\|_{\mathcal{F}}^{2} \\
\text { s. t. }\left\|\mathbf{s}_{i_{c}}\right\|_{0}=N_{c}, \\
{\left[\mathbf{R}_{s}\right]_{n, n}=\gamma \forall n}
\end{gathered}
$$

Here $\mathbf{P}_{d}$ is scaled to match the transmit power. The optimization involves a quartic problem [22], [33] under finite alphabet, uniform power and zero norm constraints, which is in general hard to solve. When $N_{0}=N_{T} N_{R}$, noting $d_{T}=N_{R} \lambda / 2$, it can be deduced that $\mathbf{A}_{T}=\sqrt{N_{T}} \mathbf{F}$, where $\mathbf{F}$ in the $N_{T} \times N_{T}$ unitary DFT matrix. It then follows from (24) that,

$$
\mathbf{P}=N_{T} \mathbf{F}^{H} \mathbf{R}_{s} \mathbf{F} .
$$

The design problem then becomes,

$$
\begin{gathered}
\min _{\mathbf{S} \in \Omega_{0}^{N_{T} \times I_{c}}}\left\|\mathbf{P}_{d}-N_{T} \mathbf{F}^{H} \mathbf{R}_{s} \mathbf{F}\right\|_{\mathcal{F}}^{2} \\
\text { s. t. }\left\|\mathbf{s}_{i_{c}}\right\|_{0}=N_{c}, \\
{\left[\mathbf{R}_{s}\right]_{n, n}=\gamma \forall n}
\end{gathered} .
$$

Incorporation of additional structure to $\mathbf{S}$ leads to tractable, albeit sub-optimal, solutions of (28); these are further discussed in the sequel. Henceforth, $\mathbf{P}_{d}, \mathbf{P}$ will be referred to as desired and generated cross-correlation beampatterns respectively.

\section{B. Block Circulant Decomposition of Signal Covariance}

The goal of the Block Circulant Decomposition (BCD) approach is to construct $\mathbf{S}$ such that the off-diagonal elements of $\mathbf{P}$ become zero. By virtue of having zero cross-correlation between the transmit antenna elements, the BCD approach maximizes angular resolution by spanning the largest possible virtual aperture. From (27), $\mathbf{P}$ becomes diagonal if the eigenvector matrix of $\mathbf{R}_{s}$ is the DFT matrix F. In fact,

$$
\mathbf{P}=\mathbf{A}_{T}^{H} \mathbf{R}_{s} \mathbf{A}_{T}=N_{T} \mathbf{F}^{H} \mathbf{R}_{s} \mathbf{F}=N_{T} \boldsymbol{\Lambda},
$$

if $\mathbf{R}_{s}=\mathbf{F} \mathbf{\Lambda} \mathbf{F}^{H}$ is the eigenvalue decomposition of $\mathbf{R}_{s}$, where the $\boldsymbol{\Lambda} \in \mathbb{R}^{N_{T} \times N_{T}}$ is the eigenvalue matrix with non-negative diagonal. From the definition of $\mathbf{P}_{d}$, these non-negative eigenvalues, or equivalently, the diagonal elements of $\mathbf{P}$, contain information about the sampled radiation pattern. Therefore, ensuring the construction of $\mathbf{S}$ such that its eigenvector matrix is a DFT matrix for any set of entries is particularly interesting since it simplifies the design. Circulant matrices exhibit this property [40], a fact exploited henceforth.

Let $N_{B}=\frac{\mathcal{I}_{c}}{N_{T}}$ be an integer and $\mathbf{B}_{b} \in \Omega_{0}^{N_{T} \times N_{T}}, b=1$, $2, \ldots, N_{B}$ be a circulant matrix with entries drawn from $\Omega_{0}$. Further, let the signal matrix, $\mathbf{S}$, be constructed using $\mathbf{B}_{b}$ as,

$$
\begin{aligned}
\mathbf{S} & =\left(\mathbf{s}_{1}, \ldots, \mathbf{s}_{i_{c}}, \ldots, \mathbf{s}_{\mathcal{I}_{c}}\right)=\left(\mathbf{B}_{1}, \ldots, \mathbf{B}_{b}, \ldots, \mathbf{B}_{N_{B}}\right) \\
\mathbf{B}_{b} & =\mathbf{F} \mathbf{\Psi}_{b} \mathbf{F}^{H},
\end{aligned}
$$

where, $\boldsymbol{\Psi}_{b}$ is the eigenvalue matrix of $\mathbf{B}_{b}$ [40]. The structure of $\mathbf{S}$, comprising blocks of circulant matrices, leads to the terminology BCD. It can be shown that the $\mathbf{S}$ generated from (30) leads to a circulant Hermitian $\mathbf{R}_{s}$,

$$
\mathbf{R}_{s}=\sum_{b=1}^{N_{B}} \mathbf{B}_{b} \mathbf{B}_{b}^{H}=\mathbf{F}\left(\sum_{b=1}^{N_{B}} \boldsymbol{\Psi}_{b} \mathbf{\Psi}_{b}^{H}\right) \mathbf{F}^{H}=\mathbf{F} \boldsymbol{\Lambda} \mathbf{F}^{H} .
$$

From (31) and earlier discussions on the off-diagonal elements of $\mathbf{P}$, it follows that the BCD based design in (30) provides an attractive framework for the sampled cross-correlation optimization. Further, the resulting circulant $\mathbf{R}_{s}$ implicitly leads to an uniform power transmission from the transmit antenna elements; this ensures the satisfaction of the uniform element power constraint as well.

\section{Beampattern Design With BCD}

Since each $\mathbf{B}_{b}$ is circulant, it is completely defined by its first column, denoted here as, $\mathbf{c}_{b} \in \Omega_{0}^{N_{T} \times 1}$ [40]. Hence, $\mathbf{S}$ in (30) is parametrized by $N_{B}$ column vectors, $\left\{\mathbf{c}_{b}\right\} \in \Omega_{0}^{N_{T} \times 1}$, where the $b$ denotes the index of the $b$-th block. Since the eigenvalues of the $b$-th block circulant matrix $\mathbf{B}_{b}$ are given by the the DFT of $\mathbf{c}_{b}$, i. e., $\boldsymbol{\Psi}_{b}=\operatorname{diag}\left(\mathbf{F} \mathbf{c}_{b}\right)$, the eigenvalues of $\mathbf{R}_{s}$ are given by actual radiation pattern vector $\mathbf{p} \in \mathbb{R}_{+}^{N_{T} \times 1}$,

$$
\mathbf{p}=\operatorname{diag}(\boldsymbol{\Lambda})=\sum_{b=1}^{N_{B}}\left(\mathbf{F}^{H} \mathbf{c}_{b}\right) \circ\left(\mathbf{F}^{H} \mathbf{c}_{b}\right)^{*} .
$$

Since only the diagonal elements of the optimization problem (26) contribute to the cost and the uniform power constraint is inherently satisfied by a $\mathrm{BCD}$, the optimization problem under 
the block-circulant construction simplifies to,

$$
\begin{aligned}
\min _{\mathbf{c}_{b} \in \Omega_{0}^{N_{T^{\times 1}}} \forall b} & \left\|\mathbf{p}_{d}-\sum_{b=1}^{N_{B}}\left(\mathbf{F}^{H} \mathbf{c}_{b}\right) \circ\left(\mathbf{F}^{H} \mathbf{c}_{b}\right)^{*}\right\|_{2}^{2} \\
\text { s. t. } & \left\|\mathbf{c}_{b}\right\|_{0}=N_{c} \forall b .
\end{aligned}
$$

The BCD provides a suitable method for obtaining good crosscorrelation beampattern properties for ULA under the uniform power constraint. It can be seen as a framework for enabling the ULA virtual MIMO paradigm. Before discussing the solution to the optimization in (33) in the sequel, we briefly relate the proposed metric to the relevant literature.

\section{Relationship to Relevant Prior-Art on Waveform Design}

We briefly relate our formulation to two relevant references: (i) beampattern matching with simultaneous cross-correlation optimization in [22], and (2) DFT based beampattern matching in [31]. Towards this, (26) can be reformulated as $\min _{\mathbf{S} \in \Omega_{0}^{N_{T} \times \mathcal{I}_{c}}} \sum_{l=1}^{N_{T}}\left|p_{d}\left(\phi_{l}, \phi_{l}\right)-\mathbf{a}_{T}^{H}\left(\phi_{l}\right) \mathbf{R}_{s} \mathbf{a}_{T}\left(\phi_{l}\right)\right|^{2}+$ $2 \sum_{\kappa=1}^{N_{T}-1} \sum_{m=\kappa+1}^{N_{T}}\left|\mathbf{a}_{T}^{H}\left(\phi_{m}\right) \mathbf{R}_{s} \mathbf{a}_{T}\left(\phi_{\kappa}\right)\right|^{2} \quad$ subject to $\left\|\mathbf{s}_{i_{c}}\right\|_{0}=N_{c}, \quad\left[\mathbf{R}_{s}\right]_{n, n}=N_{c} N_{T} N_{B} \forall n . \quad$ This expression is similar to equation (19) of [22] with specific weights $w_{c}, w_{l}$. However, notable differences include

- Generalized multiplexing and discrete phase constraints are not used in [22]. Further, we optimize the transmit waveform $\mathbf{S}$ directly while only $\mathbf{R}_{s}$ is optimized in [22]. Furthermore, the optimization of the beam-pattern scaling factor $\alpha$ in [22], is not needed since the pre-determined constraint on $\mathbf{S}$ readily provides the power level of the beampattern.

- In [22], angle-Doppler coupling is not considered, while the topic is dealt in Section V.

- The target index $\kappa$ in our approach is limited to a uniform grid of $N_{T}$ samples; we will demonstrate its negligible impact through simulations. On the other hand, the index $l$ in [22] represents a fine beampattern grid of $L>>N_{T}$ samples. Our BCD approach only requires $N_{T}$ uniform samples of the beampattern and simplifies the optimization problem.

On the other hand, [31]. similar to our work, (i) imposes conditions on the sequence length, (ii) requires only $N_{T}$ uniform samples of the transmit radiation pattern, (iii) imposes a (Toeplitz) structure to the covariance matrix and (iv) offers a closed-form design. However, key differences include:

- Cross-correlation is not considered in the design of [31], thereby making it unsuitable for a virtual MIMO paradigm

- General multiplexing constraint and discrete phase modulation with arbitrary number of phase stages is not considered in [31]. In fact, the transmitted signal is restricted to a $N_{T}$ unique phase stages in [31] when assuming a 0,1 pattern for desired beampattern; this is not the case in our study. Additionally, the sequence length in [31] is limited to $N_{T}$, while it can be a multiple of $N_{T}$ in our setting, thereby offering higher degrees of design freedom.
- In [31], angle-Doppler coupling is not considered, while the topic is dealt in Section V.

- Our approach benefits from lower complexity, due to a circulant structure of $\mathbf{R}_{s}$, unlike the Toeplitz structure based design in [31]. However, the circulant structure of our approach ensures good cross-correlation properties.

The differences between our approach and [22] and [31] are discussed in more detail through simulations.

\section{DiCTIONARY BASED OPTIMIZATION FRAMEWORK}

The finite alphabet constraint problem (general multiplexing with subsequent discrete unimodular signal design) in (33) needs additional operations towards easing the optimization process. This section provides a dictionary based framework to transform (33) into a well-known class of $l_{1}$-norm minimization (convex) problems. The focus herein is on exploiting the signal structure to generate the dictionary efficiently. In addition to the dictionary generation in the general set-up, special cases from [37] and [23] are presented wherein the dictionary generation is simplified and (33) is solved in closed form. Thus, the proposed framework extends the works [37] and [23] to the general case of having an arbitrary number of channels and phase stages.

\section{A. Optimization Framework for Signal Design}

The solution considered for the optimization in (33) is based on a dictionary approach. Accordingly, the designed radiation pattern $\mathbf{p}$ can be written in terms of $N_{D}$ distinct atoms, where the $i$-th atom and the corresponding coefficient are denoted by $\mathbf{q}_{i} \in \mathbb{R}_{+}^{N_{T} \times 1}$ and $v_{i} \in \mathbb{Z}_{+}$, respectively, thereby leading to,

$$
\mathbf{p}=N_{T} \sum_{i=1}^{N_{D}} \mathbf{q}_{i} v_{i}=N_{T} \mathbf{Q} \mathbf{v}
$$

Here, $\mathbf{Q} \in \mathbb{R}_{+}^{N_{T} \times N_{D}}$ denotes the dictionary matrix and $\mathbf{v} \in$ $\mathbb{Z}_{+}^{N_{D} \times 1}$ denotes the atom coefficient vector. While there is no restriction on the number of atoms, to ensure that (34) represents (32) adequately and efficiently, the following constraints are imposed,

1) Each atom is generated using an arbitrary (as yet) transmit array excitation $\mathbf{c}_{i} \in \Omega_{0}^{N_{T} \times 1},\left\|\mathbf{c}_{i}\right\|_{0}=N_{c}$ as,

$$
\mathbf{q}_{i}=\left(\mathbf{F}^{H} \mathbf{c}_{i}\right) \circ\left(\mathbf{F}^{H} \mathbf{c}_{i}\right)^{*}, i \in\left[1, N_{D}\right]
$$

where $\quad \Omega_{0}=\{0\} \cup\left\{1, \exp \left(j \frac{2 \pi}{N_{p}}\right) \cdots \exp \left(j \frac{2 \pi\left(N_{p}-1\right)}{N_{p}}\right)\right\}$ contains the possible phase modulations and zero (for multiplexing). This enables a structural similarity of (34) to (32) and renders $\mathbf{q}_{i}$ to be non-negative.

2) Note that (32) requires no more than $N_{B}$ vectors, $\left\{\mathbf{c}_{*}\right\}$, while (35) considers $N_{D}$ vectors. To ensure consistency, scaling factors $v_{i}$ have to be non-negative integers and satisfy the $l_{1}$-norm constraint, $\|\mathbf{v}\|_{1}=N_{B}$. These two constraints ensure no more than $N_{B}$ terms in the summation of (34) including repetitions of $\mathbf{q}_{i}$, even when $N_{D}>N_{B}$. In particular, this ensures $\sum_{i=1}^{N_{D}} \mathbf{q}_{i} v_{i}=\sum_{i=1}^{N_{B}} \mathbf{q}_{\phi(i)}$, where $\phi(i) \in\left[1, N_{D}\right]$ is the mapping set and $\mathbf{c}_{\phi(i)}$ is used to construct $\mathbf{v}_{i}$ circulant blocks. 
The optimization problem (33) can be reformulated as,

$$
\begin{gathered}
\min _{\mathbf{v} \in \mathbb{Z}_{+}^{N^{\times} \times 1}}\left\|\mathbf{p}_{d}-N_{T} \mathbf{Q v}\right\|_{2}^{2} \\
\text { s. t. }\|\mathbf{v}\|_{1}=N_{B} .
\end{gathered}
$$

The dictionary $\mathbf{Q}$ can be designed offline using arbitrary $\mathbf{c}_{i} \in$ $\Omega_{0}^{N_{T} \times 1}$ with $\left\|\mathbf{c}_{i}\right\|_{0}=N_{c}$ from (35); this implicitly satisfies the multiplexing and discrete phase modulation constraints. The problem (36) is non-convex due to the non-negative integer value constraint of $\mathbf{v} \in \mathbb{Z}_{+}^{N_{D} \times 1}$. It can be simplified if $\mathbf{v}$ is relaxed to $\hat{\mathbf{v}} \in \mathbb{R}^{N_{D} \times 1}$, a vector of non-negative reals. However, in order to relate the coefficient vector to the column vectors $\left\{\mathbf{c}_{b}\right\}$, a subsequent rounding to the nearest integer is necessary.

The problem in (36) can be reformulated as,

$$
\begin{aligned}
\min _{\hat{\mathbf{v}} \in \mathbb{R}^{N} \times 1} & \|\hat{\mathbf{v}}\|_{0} \\
\text { s. t. } & \left\|\mathbf{p}_{d}-N_{T} \mathbf{Q} \hat{\mathbf{v}}\right\|_{2}^{2} \leq \epsilon \\
& \hat{\mathbf{v}} \geq \mathbf{0} .
\end{aligned}
$$

where $\epsilon>0$ is a given design constraint and the relationship between the real $\hat{\mathbf{v}}$ and the integer $\mathbf{v}$ is defined by the rounding function $\Xi(x)=\max \{k \in \mathbb{Z} \mid k \leq x+0.5\}$, as,

$$
\mathbf{v}=\Xi\left(\frac{\hat{\mathbf{v}}}{\|\hat{\mathbf{v}}\|_{1}} N_{B}\right)
$$

The aforementioned construction of $\mathbf{v}$ closely approximates the requirement $\|\mathbf{v}\|_{1}=N_{B}$ in (36) and the slight deviation, if any, arises due to the rounding. In this context, it is clear that a good rounding property, and hence closer approximation of $l_{1}$ norm constraint, is achieved for a sparse solution of the $\hat{\mathbf{v}} \in \mathbb{R}^{N_{D} \times 1}$. This motivates the $l_{0}$ objective in (37). In case $\|\mathbf{v}\|_{1} \neq N_{B}$, it is adjusted to $N_{B}$ by increasing/ decreasing randomly chosen entries of $\mathbf{v}$. While this is indeed sub-optimal, it is easy to implement with, possibly, limited performance degradation.

1) Robust Design Criteria: Since the desired beampattern can be only approximated due to physical limitations, a suitable real non-negative error $\epsilon \in \mathbb{R}_{+}$has to be considered to ensure robust design. Equation (37) can be relaxed to a convex problem by the well known $l_{1}$-norm relaxation [41],

$$
\begin{gathered}
\min _{\hat{\mathbf{v}} \in \mathbb{R}^{N_{D} \times 1}, \epsilon \in \mathbb{R}}\|\hat{\mathbf{v}}\|_{1}+\eta \epsilon \\
\text { s. t. }\left\|\mathbf{p}_{d}-N_{T} \mathbf{Q} \hat{\mathbf{v}}\right\|_{2}^{2} \leq \epsilon \\
\hat{\mathbf{v}} \geq \mathbf{0} \\
\epsilon \geq 0 .
\end{gathered}
$$

The optimization tuning parameter $\eta \in \mathbb{R}_{+}$takes into account the trade-off between sparsity of the coefficient vector and the beampattern error. In other words, this trade-off deals with the trade-off between rounding error and beam pattern error. The optimization problem (39) is convex and can be easily solved in polynomial time using well-known solvers. Together with the BCD approach, the optimization problem (39) provides a crosscorrelation beampattern design framework for any waveform alphabet. Table I summarizes the proposed BCD framework for beampattern design.
TABLE I

Algorithm for General Phase Modulation and Multiplex SEQUENCE DESIGN

Step 0: Initialize desired beampattern $\mathbf{p}_{d}$, vectors $\left\{\mathbf{c}_{b}\right\}$ s, dictionary $\mathbf{Q}$

Step 1: Calculate $\hat{\mathbf{v}}$ solving the problem in (39)

Step 2: Quantize $\hat{\mathbf{v}}$ into $\mathbf{v}$ using (38)

Step 3: Randomly update an element of $\mathbf{v}$ to ensure $\|\mathbf{v}\|_{1}=N_{B}$. For each index $i$ with $\mathbf{v}_{i}>0$, obtain $\mathbf{c}_{i}$ used to generate corresponding $\mathbf{q}_{i}$

Step 4: $\mathbf{S}$ is constructed by circularly shifting (across columns) each $\mathbf{c}_{i}$ from Step $3 N_{T} \mathbf{v}_{i}$ times, i.e., $\mathbf{v}_{i}$. circulant blocks constructed from $\mathbf{c}_{i}$

The error in the signal design framework is justified by the rounding error as well as by the design of a suitable dictionary matrix $\mathbf{Q}$. The design of the dictionary $\mathbf{Q}$ is tackled for particular modulation sets in the sequel, whereas the rounding error is investigated through simulations.

2) Computational Complexity: In the most general case, the problem in (39) can be treated as a non-negative Least squares problem and solved using interior point methods, which are of the order $O\left(N_{D}^{2} N_{T}\right)-O\left(N_{D}^{3}\right)$ [42]. However, with minor modifications to incorporate the non-negative constraints, the problem in (39) is similar to the Basis Pursuit Denoising (BPDN) [43]. Several fast algorithms from the unconstrained BPDN framework can be adapted to incorporate the non-negative requirement to solve this problem faster [43]. The complexity depends on the solver and the problem setting; as an example, the detailed complexity for the In-Crowd algorithm is provided in [43].

\section{B. Discrete Phase Unimodular Design}

The unimodular design for $\mathbf{S}$ provides the best power efficiency [11] and is therefore of great interest. Further, the discrete phase stages are an attractive practical design constraint. For the particular case with identical number of transmit antennas, channels and phase stages $N_{T}=N_{P}=N_{c}$, the problem in (39) can be solved in closed form, due to an appropriate choice of dictionary matrix [23].

In particular, $\mathbf{c}_{i}$ is chosen from the Fourier bases as,

$$
\left[\mathbf{c}_{i}\right]_{n}=\exp \left(j \frac{2 \pi}{N_{T}}(n-1)(i-1)\right), i \in\left[1, N_{B}\right] .
$$

Using (35), the resulting dictionary simplifies to,

$$
\mathbf{Q}=N_{T} \mathbf{I}_{N_{T}} .
$$

This choice is only satisfied for the case of $N_{T}=N_{P}=N_{c}$.

1) Design Algorithm: Since the dictionary matrix reduces to a scaled identity, it is not necessary to solve (39). Step. 1 in the signal design algorithm in Table I is replaced by a closed form, $\hat{\mathbf{v}}=\mathbf{p}_{d} / N_{T}$, (scaled desired radiation pattern) due to (41). Therefore Step 2 of the signal design algorithm directly considers the desired radiation pattern to yield,

$$
\mathbf{v}=\Xi\left(\frac{\mathbf{p}_{d}}{\left\|\mathbf{p}_{\mathbf{d}}\right\|_{1}} N_{B}\right) .
$$

The proposed signal design algorithm for discrete phase sequences is a closed form solution without any iterative steps. Performance in this scenario depends on $N_{B}$ due to their ability 
to reduce the quantization error. Performance of such designs is illustrated in the numerical results section.

\section{Two Channel QPSK Based Design}

The two channel multiplexing is of interest as it reflects the minimum number of channels needed to design a beampattern from isotropic radiators. Further, this design considers the 2D Quadrature Phase Shift Keying (QPSK) modulation, i.e., $\Omega=\{1,-1,-j, j\}$. This approach lends to a simple solution benefiting from a structured dictionary formulation.

1) Dictionary Formulation: Since two channels are switched on, only two elements of any arbitrary $\mathbf{c}_{n}$, say $i_{1}$ and $i_{2}$ with $i_{1} \neq i_{2}$ are non-zero. Hence only two column vectors of $\mathbf{F}^{H}$, denoted by $\mathbf{f}_{i_{1}}$ and $\mathbf{f}_{i_{2}}$ are of interest for when evaluating $\left(\mathbf{F}^{H} \mathbf{c}_{n}\right) \circ\left(\mathbf{F}^{H} \mathbf{c}_{n}\right)$. With $\mathbf{q}_{n}$ being the atom generated from this $\mathbf{c}_{n}$, it then follows that,

$$
\begin{aligned}
\mathbf{q}_{n} & =\left(\mathbf{F}^{H} \mathbf{c}_{n}\right) \circ\left(\mathbf{F}^{H} \mathbf{c}_{n}\right)^{*} \\
& =2 \mathbf{1}_{N_{T}}+2 \Re\left\{\left[\mathbf{c}_{n}\right]_{i_{1}}\left[\mathbf{c}_{n}\right]_{i_{2}}^{*}\left(\mathbf{f}_{i_{1}} \circ \mathbf{f}_{i_{2}}^{*}\right)\right\},
\end{aligned}
$$

where $\mathbf{1}_{N_{T}}$ in a column of $N_{T}$ ones. Since $N_{c}=2$, the $m$-th element of $\mathbf{q}_{n}$, denoted as $\left[\mathbf{q}_{n}\right]_{m}$, takes the form,

$\left[\mathbf{q}_{n}\right]_{m}=2+2 \cos \left(\frac{2 \pi}{N_{T}}\left(i_{1}-i_{2}\right)(m-1)+\left(\varphi_{n, i_{1}}-\varphi_{n, i_{2}}\right)\right)$,

where, $\varphi_{n, i_{1}}=\angle\left[\mathbf{c}_{n}\right]_{i_{1}}$ and $\varphi_{n, i_{2}}=\angle\left[\mathbf{c}_{n}\right]_{i_{2}}$ reflect the phase information of the $i_{1}$-th and $i_{2}$-th vector entry of $\mathbf{c}_{n}$. It can be seen from (44) that each $\mathbf{q}_{n}$ is characterized by two parameters

1) The relative distance between excited antenna elements, $i_{1}-i_{2}$, determines the frequency of cosine function. Due to the limited transmit antenna array size, there can only be $N_{T}-1$ different frequencies, thereby leading to $N_{T}-1$ linearly independent functions.

2) Due to the QPSK modulation, there are 4 possible phase difference per frequency given by $\varphi_{n, i_{2}}-\varphi_{n, i_{1}} \in$ $\{0, \pi / 2, \pi, 3 \pi / 2\}$ irrespective of $n, i_{1}, i_{2}$ for each frequency. The relative phase of the excited antenna elements $\varphi_{n, i_{2}}-\varphi_{n, i_{1}}$ determines if a positive/ negative sine or cosine function appears in the atom.

Hence, the number of distinct atoms is $4\left(N_{T}-1\right)$. Using $2 \cos ^{2}\left(\frac{x}{2}\right)=1+\cos (x)$, equation (44), $\Delta \varphi_{l}=(l-1) \pi / 2$ and $n=4(k-1)+l, k \in\left[1, N_{T}-1\right], l \in[1,4]$, the atoms are positive and take the form,

$$
\left[\mathbf{q}_{n}\right]_{m}=4 \cos ^{2}\left(\frac{\pi}{N_{T}}(m-1) k+\frac{\Delta \varphi_{l}}{2}\right) .
$$

2) Reformulation of Optimization: With the dictionary matrix defined, the steps in Table I can be pursued towards signal design. However, the closed-form expression for $\mathbf{q}_{n}$ can be exploited to avoid Step 1 as in Section IV-B. Towards this, at first, a reformulation $\mathbf{q}_{n}$ in (44) using $\cos (x+$ $y)=\cos (x) \cos (y)-\sin (x) \sin (y)$ is undertaken. Letting, $n=$ $4(k-1)+l, l \in[1,4], k \in\left[1, N_{T}-1\right]$, this leads to

$$
\left[\mathbf{q}_{n}\right]_{m}=2+2\left(\cos \left(\Delta \varphi_{l}\right) \cos \left(\frac{2 \pi}{N_{T}}(m-1) k\right)\right.
$$

TABLE II

SignAl MATRIX GENERATION PRINCIPLE FOR QPSK

\begin{tabular}{c|c|c|c}
\hline $\begin{array}{c}k \text {-th Fourier coefficient } \\
k=i_{1}-i_{2}\end{array}$ & {$\left[\mathbf{c}_{n}\right]_{i_{1}}\left[\mathbf{c}_{n}\right]_{i_{2}}^{*}$} & $\Delta \varphi_{l}$ & Number of $\mathbf{c}_{n}$ \\
\hline \hline$\left[\mathbf{a}_{c}\right]_{k}>0$ & 0 & 0 & {$\left[\hat{\mathbf{a}}_{c}\right]_{k}$} \\
{$\left[\mathbf{a}_{s}\right]_{k}>0$} & $j$ & $\pi / 2$ & {$\left[\hat{\mathbf{a}}_{s}\right]_{k}$} \\
{$\left[\mathbf{a}_{c}\right]_{k}<0$} & -1 & $\pi$ & {$\left[\hat{\mathbf{a}}_{c}\right]_{k}$} \\
{$\left[\mathbf{a}_{s}\right]_{k}<0$} & $-j$ & $3 \pi / 2$ & {$\left[\hat{\mathbf{a}}_{s}\right]_{k}$} \\
\hline
\end{tabular}

$$
\left.-\sin \left(\Delta \varphi_{l}\right) \sin \left(\frac{2 \pi}{N_{T}}(m-1) k\right)\right) .
$$

With the $4\left(N_{T}-1\right)$ atoms defined, the $n$-th element of the generated radiation pattern in (34) takes the following form,

$$
\begin{aligned}
{[\mathbf{p}]_{n}=} & 2 N_{T} N_{B}+2 N_{T} \sum_{k=1}^{N_{T}-1}\left[\left[\mathbf{a}_{c}\right]_{k} \cos \left(\frac{2 \pi}{N_{T}}(n-1) k\right)\right. \\
& \left.+\left[\mathbf{a}_{s}\right]_{k} \sin \left(\frac{2 \pi}{N_{T}}(n-1) k\right)\right]
\end{aligned}
$$

where $\mathbf{a}_{c}, \mathbf{a}_{s} \in \mathbb{R}^{\left(N_{T}-1\right) \times 1}$ are coefficients of the sine/ cosine terms. Since these sine/ cosine terms are not the atoms per-se (given in (44)), $\mathbf{a}_{c}, \mathbf{a}_{s}$ are different from $\mathbf{v}$ of (34).

The expression in (47) is similar to the Fourier series. Hence, the elements of $\mathbf{a}_{c}, \mathbf{a}_{s}$ can be obtained analogously to Fourier coefficients as,

$$
\begin{gathered}
{\left[\mathbf{a}_{c}\right]_{k}=\frac{1}{2 N_{T}} \sum_{n=1}^{N_{T}-1}\left(\left[\mathbf{p}_{d}\right]_{n}-2 N_{T} N_{B}\right) \cos \left(\frac{2 \pi}{N_{T}} k n\right)} \\
{\left[\mathbf{a}_{s}\right]_{k}=\frac{1}{2 N_{T}} \sum_{n=1}^{N_{T}-1}\left(\left[\mathbf{p}_{d}\right]_{n}-2 N_{T} N_{B}\right) \sin \left(\frac{2 \pi}{N_{T}} k n\right) .}
\end{gathered}
$$

The Fourier coefficients have to be mapped to the actual transmit signal matrix $\mathbf{S}$. The first step towards this is to round these coefficients to a positive integer as,

$$
\begin{aligned}
{\left[\hat{\mathbf{a}}_{c}\right]_{k} } & =\Xi\left(\frac{\left|\left[\mathbf{a}_{c}\right]_{k}\right|}{\left(\left|\mathbf{a}_{c}\right|_{1}+\left|\mathbf{a}_{s}\right|_{1}\right)} N_{B}\right) \\
{\left[\hat{\mathbf{a}}_{s}\right]_{k} } & =\Xi\left(\frac{\left|\left[\mathbf{a}_{s}\right]_{k}\right|}{\left(\left|\mathbf{a}_{c}\right|_{1}+\left|\mathbf{a}_{s}\right|_{1}\right)} N_{B}\right) .
\end{aligned}
$$

The function $\Xi$ denotes the rounding function. Further, the following relationship has to be satisfied,

$$
N_{B}=\left(\left|\hat{\mathbf{a}}_{c}\right|_{1}+\left|\hat{\mathbf{a}}_{s}\right|_{1}\right) .
$$

The rounding in (50) and (51) meets the requirement in (52) closely. As in the previous discussions, a randomly chosen coefficient is increased/ decreased to satisfy the requirement.

3) Signal Matrix Generation: Table II illustrates the generation of the BCD blocks from the Fourier coefficients $\mathbf{a}_{c}, \mathbf{a}_{s}$. As an example, if $\left[\mathbf{a}_{s}\right]_{k}>0$, it follows from (46), (47) that the frequency of the sine wave, and hence the distance between non-zero elements, $\left[\mathbf{c}_{n}\right]_{i_{1}},\left[\mathbf{c}_{n}\right]_{i_{2}}$ of $\mathbf{c}_{n}$ is $k$. Due to the BCD, the actual choice $i_{1}, i_{2}$ does not matter as long as $\left|i_{1}-i_{2}\right|=k$ Further, the phase difference between the excitation need to be $\pi / 2$, kindly refer to (46), (47). Further, the determined $\mathbf{c}_{n}$, is used 
TABLE III

ALGORITHM FOR Two CHANNEL TDM QPSK MODULATION

\begin{tabular}{l}
\hline Step 0: Initialize desired beampattern $\mathbf{p}_{d}$ \\
\hline Step 1: Calculate Fourier coefficients using (48) and (49)) \\
Step 2: Obtain Fourier coefficients as in (50) and (51)); \\
increase/ decrease one of them to satisfy (52) \\
Step 3: Map the Fourier coefficients to $\mathbf{c}_{n}$ as illustrated in \\
Table II. The number of repetitions of $\mathbf{c}_{n}$ are determined. \\
Step 4: Using $\mathbf{c}_{n}$ and its repetitions from Step3, use BCD to obtain $\mathbf{S}$. \\
\hline
\end{tabular}

in $\left[\hat{\mathbf{a}}_{c}\right]_{k}$ blocks. New excitation vectors are similarly determined for all other cases.

It can be seen from (44) that only the phase difference $\Delta \varphi$ counts, leading to a degree of freedom in the design procedure. A way is to set $\left[\mathbf{c}_{n}\right]_{i_{1}}$ equal to one and determine $\left[\mathbf{c}_{n}\right]_{i_{2}}$ accordingly. Another way is to select $\left[\mathbf{c}_{n}\right]_{i_{1}}$ randomly out of the given set and determine $\left[\mathbf{c}_{n}\right]_{i_{2}}$ accordingly. Table III shows the signal design algorithm for the two channel QPSK modulation. This algorithm provides a low complexity, possibly sub-optimal, solution to (33) under the aforementioned constraints. The computations involve Fourier transform evaluations and standard algebraic operations, both not exceeding $O\left(N_{T}^{2}\right)$.

In contrast to the closed form solution in [31], [32] and [17] the proposed approach takes the virtual MIMO configuration into account and further considers finite alphabet design constraints. The presented two channel QPSK approach can be seen as a special case of the proposed framework where (39) is solved for the dictionary in (44). Further, in contrast to the discrete phase approach, the dictionary size is larger than the number of transmit antennas $N_{D} \geq N_{T}$, but the interpretation using Fourier series enables a closed-form solution.

\section{DOPPler Coupling ANALYsis}

The signal design described earlier omitted Doppler, thereby limiting its application only to stationary targets. Additionally, it is known from TDM MIMO that the pulse-by-pulse antenna switching leads to angle-Doppler coupling. These merit the consideration of Doppler and the potential coupling issues. A first step is the investigation of the MF output in the presence of Doppler.

\section{A. Angle-Doppler Coupling in Generalized TDM MIMO}

The angle-Doppler matched filter output is given in (12). The second term of (12) captures the angle-Doppler response; denoting this for the target $\kappa$ as $\mu_{A D, \kappa}$, using $\mathcal{I}_{c}=N_{B} N_{T}$, $\Delta \omega_{\kappa}=\omega_{M}-\omega_{\kappa}$ and the construction in (30), it follows that,

$$
\begin{aligned}
& \mu_{A D, \kappa}= \\
& =\left|\sum_{b=0}^{N_{B}-1} e^{j \Delta \omega_{\kappa} N_{T} b} \sum_{p=1}^{N_{T}} \mathbf{a}_{T, M}^{H} \mathbf{s}_{N_{T} b+p} \mathbf{s}_{N_{T} b+p}^{H} \mathbf{a}_{T, \kappa} e^{j \Delta \omega_{\kappa} p}\right|^{2}
\end{aligned}
$$

Note that the sum over $\mathcal{I}_{c}$ in (12) is replaced by a sum over $b$ and $p$. The outer sum represents the sum over blocks, denoted by the block index $b$ while the inner sum is over the columns within each block (denoted by $p$ ). Since the columns within the block $\mathbf{B}_{b+1}$, i.e, $\mathbf{s}_{N_{T} b+p}, p=1, \ldots, N_{T}$, are circular shifted versions of $\mathbf{c}_{b+1}$, it can be shown using the shift theorem of the Fourier transform that $\mathbf{a}_{T, M}^{H} \mathbf{s}_{N_{T} b+k}=e^{-j k_{0} \sin \phi_{M} p} \mathbf{a}_{T, M}^{H} \mathbf{c}_{b+1}$. Using a similar expression for $\mathbf{a}_{T, \kappa}^{H} \mathbf{s}_{N_{T} b+k}$, and denoting $\Delta k_{\kappa}=$ $k_{0}\left(\sin \left(\phi_{M}\right)-\sin \left(\phi_{\kappa}\right)\right)$ to be the relative angle response between the MF output $\phi_{M}$ and the actual target position $\phi_{\kappa}$, it follows that,

$$
\begin{aligned}
\mu_{A D, \kappa} & =\left|\sum_{p=1}^{N_{T}} e^{j \Delta \omega_{\kappa} p} e^{-j \Delta k_{\kappa} p}\right|^{2} \\
& \times\left|\sum_{b=0}^{N_{B}-1} \mathbf{a}_{T, M}^{H} \mathbf{c}_{b+1} \mathbf{c}_{b+1}^{H} \mathbf{a}_{T, \kappa} e^{j \Delta \omega_{\kappa} N_{T} b}\right|^{2} .
\end{aligned}
$$

Further, the term $e^{-j \Delta k_{\kappa} p}$ illustrates a linear phase center motion (PCM), which leads to a strong angle-Doppler coupling [18]. This is illustrated by the first term in (54) which leads to peaky side-lobes. Since this linear PCM is a result of the block circulant construction, modifications to this construction in (30) are warranted. In this context, it is known from [18] that the angle-Doppler coupling due to linear PCM can be overcome by signal randomization; this motivates the subsequent investigation.

The second term in (54) represents a DFT operation as well, but the spectrum is inherently $N_{T}$ periodic. This periodicity is also justified by the block circulant construction of (30). Further, it is desired that the argument of the second DFT i. e., $\mathbf{a}_{T, M}^{H} \mathbf{c}_{b} \mathbf{c}_{b}^{H} \mathbf{a}_{T, \kappa}$, coherently sums up such that a peak at $\Delta \omega_{\kappa}=0$ occurs. Since the vectors $\left\{\mathbf{c}_{b}\right\}$ are the output of the optimization algorithm and not known a priori, a conjugate symmetric condition on $\left\{\mathbf{c}_{b}\right\}$ is introduced. This simplifying condition results in $\mathbf{a}_{T, M}^{H} \mathbf{c}_{b} \mathbf{c}_{b}^{H} \mathbf{a}_{T, \kappa}$ being real for all $b$, potentially enabling coherent summation at $\Delta \omega_{\kappa}=0$.

These modifications to signal design are discussed next.

\section{B. Randomization of Signal Matrix}

1) Motivation: The angle-Doppler coupling, described by $\mu_{A D, \kappa}$ in (54), is dependent on the PCM properties of the designed transmit signal matrix. Unlike in the case of zero Doppler, this coupling is dependent on the temporal order of antenna excitation. Clearly, optimizing the temporal ordering in the design adds to its complexity; to ease the design procedure while incorporating this dependence, a stochastic model for the signal matrix is assumed henceforth. Such an approach enables the angle-Doppler coupling mitigation ability to the low complexity BCD signal design paradigm.

2) Implementation and Modelling: A statistical approach to alter the phase centre, while considering the $\mathrm{BCD}$, is pursued by randomly permuting the columns of $\mathbf{S}$. To analyze this situation, we model the resulting $\mathbf{s}_{p}$ to be random vectors which are independent for different $p$. This approach corresponds to the view that each vector $\mathbf{s}_{p}$ is obtained in a two-step process: firstly, $\mathbf{a}_{b}$ from a given set is chosen (uniformly) randomly and (b) the chosen vector is circularly shifted randomly (uniformly). This simple stochastic model augurs well with the random column 
permutation of the signal matrix. Hence, it will henceforth be used to model the random column permutation of $\mathbf{S}$. The impact of this modelling is highlighted in the next section.

3) Expected Matched Filter Output: Analogous to the stochastic approach in [18], the ensemble average of (12) over signal distribution is considered. Focussing on the coupling, the resulting expected angle-Doppler coupling, $\bar{\mu}_{A D, \kappa} \in \mathbb{R}_{+}$, at the output of a matched filter in (12) takes the form,

$\bar{\mu}_{A D, \kappa}=E\left\{\left|\mathbf{a}_{T, M}^{H}\left(\sum_{i_{c}=1}^{\mathcal{I}_{c}} \mathbf{s}_{i_{c}} \mathbf{s}_{i_{c}}^{H} e^{-j \Delta \omega_{\kappa} T_{c} i_{c}}\right) \mathbf{a}_{T, \kappa}\right|^{2}\right\}$

where the expectation is over the distribution of $\mathbf{s}_{i_{c}}$. Letting $z_{p}=\mathbf{a}_{T, M}^{H} \mathbf{s}_{p} \mathbf{s}_{p}^{H} \mathbf{a}_{T, \kappa}^{H}$, (55) can be simplified to

$$
\bar{\mu}_{A D, \kappa}=\sum_{i_{1}=1}^{\mathcal{I}_{c}} \sum_{i_{2}=1}^{\mathcal{I}_{c}} E\left(z_{i_{1}} z_{i_{2}}^{H}\right) e^{-j \Delta \omega_{\kappa} T_{c}\left(i_{1}-i_{2}\right)} .
$$

Using the modelling assumption that $\mathbf{s}_{k}$ and $\mathbf{s}_{l}$ are independent for $k \neq l, \mathbf{R}_{s}=E\left[\mathbf{s}_{p} \mathbf{s}_{p}^{H}\right], \forall p$ (identically distributed), and $z_{p}=\mathbf{a}_{T, M}^{H} \mathbf{s}_{p} \mathbf{s}_{p}^{H} \mathbf{a}_{T, \kappa}^{H}$, (56) can be simplified as,

$$
E\left(z_{i_{1}} z_{i_{2}}^{H}\right)=\left\{\begin{array}{l}
\left|\mathbf{a}_{T, M}^{H} \mathbf{R}_{s} \mathbf{a}_{T, \kappa}^{H}\right|^{2}, i_{1} \neq i_{2} \\
E\left[\left|\mathbf{a}_{T, M}^{H} \mathbf{s}_{p} \mathbf{s}_{p}^{H} \mathbf{a}_{T, \kappa}^{H}\right|^{2}\right], i_{1}=i_{2}=p
\end{array}\right.
$$

Using (57) in (56), it follows that,

$$
\begin{aligned}
\bar{\mu}_{A D, \kappa}= & \sum_{i_{1}=1}^{\mathcal{I}_{c}}\left[E\left[\left|\mathbf{a}_{T, M}^{H} \mathbf{s}_{i_{1}} \mathbf{s}_{i_{1}}^{H} \mathbf{a}_{T, \kappa}^{H}\right|^{2}\right]-\left|\mathbf{a}_{T, M}^{H} \mathbf{R}_{s} \mathbf{a}_{T, \kappa}^{H}\right|^{2}\right] \\
& +\sum_{i_{1}=1}^{\mathcal{I}_{c}} \sum_{i_{2}=1}^{\mathcal{I}_{c}}\left|\mathbf{a}_{T, M}^{H} \mathbf{R}_{s} \mathbf{a}_{T, \kappa}^{H}\right|^{2} e^{-j \Delta \omega_{\kappa} T_{c}\left(i_{1}-i_{2}\right)}
\end{aligned}
$$

Since $\left\{\mathbf{s}_{p}\right\}$ are identically distributed, $E\left[\left|\mathbf{a}_{T, M}^{H} \mathbf{s}_{i_{1}} \mathbf{s}_{i_{1}}^{H} \mathbf{a}_{T, \kappa}^{H}\right|^{2}\right]$ is independent of pulse index and (58) further simplifies to,

$$
\begin{aligned}
\bar{\mu}_{A D, \kappa}= & \left|\mathcal{I}_{c}\right|\left[E\left[\left|\mathbf{a}_{T, M}^{H} \mathbf{s}_{p} \mathbf{s}_{p}^{H} \mathbf{a}_{T, \kappa}^{H}\right|^{2}\right]-\left|\mathbf{a}_{T, M}^{H} \mathbf{R}_{s} \mathbf{a}_{T, \kappa}^{H}\right|^{2}\right] \\
& +\left|\mathcal{I}_{c}\right|^{2}\left|W\left(\Delta \omega_{\kappa} T_{c}\right)\right|^{2}\left|\mathbf{a}_{T, M}^{H} \mathbf{R}_{s} \mathbf{a}_{T, \kappa}\right|^{2}
\end{aligned}
$$

where, $W\left(\Delta \omega_{\kappa} T_{c}\right)$ is the frequency response of a rectangular window of length $\mathcal{I}_{c}$ evaluated at $\Delta \omega_{\kappa} T_{c}$. The expected matched filter output in (59) generalizes the white PCM approach (for classical TDM) in [18] and its interpretation is provided next.

\section{Coupling Analysis and Design Algorithm}

The expression in (59) comprises two terms, the perfect MF response related to the factor $\left|\mathcal{I}_{c}\right|^{2}$ (with peak at the target position) and the coupling term related to $\left|\mathcal{I}_{c}\right|$. It can be seen that in the limit $\left|\mathcal{I}_{c}\right| \rightarrow \infty$, the coupling term vanishes. However, the sequence length is finite in practical signal design and the coupling term persists.

The coupling term is a function of the angular domain with the Doppler cuts being strongly dependent on the angle. The coupling is also a function of the transmit signal, albeit, at a lower contribution than the other term. Therefore, the coupling term shapes the sidelobe floor according to the cross-correlation
TABLE IV

General Signal Design Algorithm

properties. Thus the randomization results in the transformation of the strong coupling (typically peaky side-lobes) in (54) to a side-lobe floor, enabling enhanced target discrimination.

The Doppler analysis completes the proposed inter-pulse signal design. Table IV shows the complete signal design framework. The algorithm is initialized by a desired radiation pattern and an offline designed dictionary matrix. As shown earlier, the dictionary matrix is crucial for the algorithm performance and its impact is further discussed through simulations. The next step is to solve the proposed convex problem in (39). If the signal is constrained to $N_{T}$ uniform phase stages with $N_{c}=N_{T}$ or to two channel QPSK modulation, the convex optimization problem can be replaced by the presented closed form solutions. The third step is the rounding of the coefficient vector and in the fourth step, a block circulant matrix is constructed; this construction accounts for the cross-correlation properties and therefore for the virtual MIMO concept. Finally the proposed randomization is exploited to address angle-Doppler coupling.

The devised BCD framework proposes an inter-pulse modulation signal design for any discrete phase modulation with a subsequent multiplexing. It enables the virtual MIMO concept, is related to PCM approaches and the angle-Doppler coupling issue is mitigated. The ensuing section highlights the performance advantages through numerical simulations.

\section{Simulation Results}

1) Simulation Set-Up: The simulations are carried out with ten transmit antennas, $N_{T}=10$, and four receive antennas, $N_{R}=4$, in a MIMO configuration as described in Section II. Unless mentioned otherwise, the number of channels and phase stages are set to six, $N_{P}=N_{c}=6$, an arbitrary number satisfying the general multiplexing paradigm $1<N_{c}<N_{T}$. The dictionary size and number of blocks are chosen to $N_{D}=500$ and $N_{B}=500$, respectively. This setup is called the standard simulation setup in the sequel. On the other hand, the paper also considers the special case of $N_{T}=N_{P}=N_{c}=10$ where the dictionary is chosen as a DFT matrix, leading to $N_{D}=N_{T}=10$; this is referred to as the discrete unimodular simulation setup. In the plots for the standard simulation setup, the legends, General Framework Conjugate Symmetric and General Framework refer to the design in Section IV-A with and without conjugate symmetric constraints on excitation. Further, the legends Unimodular and 2 Channel QPSK refer to the designs from Sections IV-B and IV-C respectively. It is further noted that utmost $5 \%$ additional increase/ decrease of 


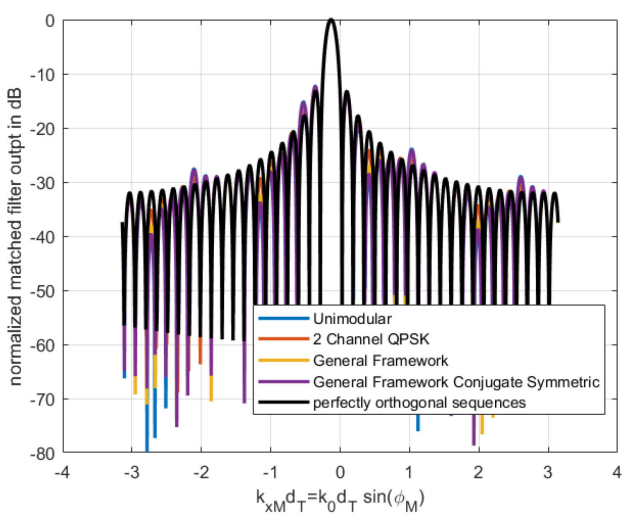

Fig. 2. Matched filter output for the angular domain. The perfectly orthogonal response and the BCD approach are almost perfectly aligned.

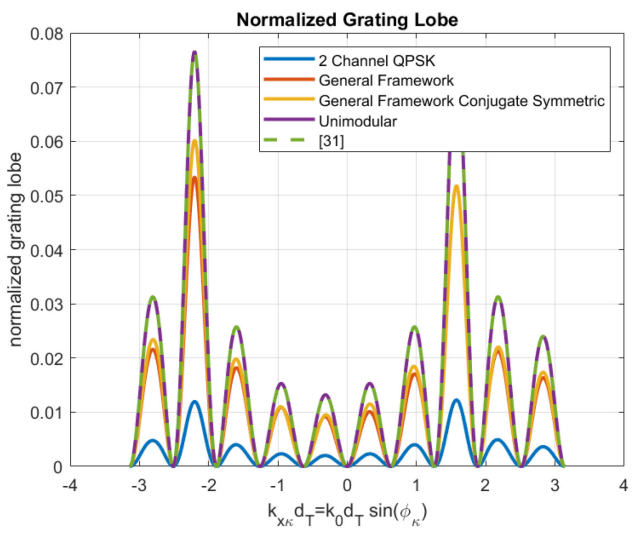

Fig. 3. Grating lobe attenuation for all possible target positions and the rectangular beampattern in figure 4 .

quantized coefficients (kindly refer Step 3 in Tables I, III, IV) is needed to satisfy the $l_{1}$ norm in the ensuing simulations. We choose $\eta=1$ for all simulations.

2) Enabling Virtual MIMO: Fig. 2 illustrates the resolution performance of different dictionary matrices. It can be seen from Fig. 2, that all approaches almost align with the perfectly orthogonal signal case where $\mathbf{R}_{s}=\gamma \mathbf{I}_{N_{T}}, \gamma>0$; this demonstrates the effectiveness of the BCD approach and its cross-correlation properties. The Mean Square Error (MSE) between perfectly orthogonal signals and the algorithm output for a randomly initialized dictionary matrix averages $-18 \mathrm{~dB}$. The MSE for the unimodular simulation setup is practically zero, which implies that the appropriate choice of the dictionary matrix is crucial to the design.

Fig. 3 illustrates the variation of cross-correlation term, $\sum_{\kappa=1}^{N_{T}-1} \sum_{m=\kappa+1}^{N_{T}}\left|\mathbf{a}_{T}^{H}\left(\phi_{m}\right) \mathbf{R}_{s} \mathbf{a}_{T}\left(\phi_{\kappa}\right)\right|^{2}$ normalized to the main lobe power, known henceforth as the normalized grating lobe, as a function of a single target position. Several configurations including [31] are plotted. The related beam shape is depicted in Fig. 4. We see that the grating lobes are perfectly attenuated at the beampattern sampling points, similar to orthogonal sequences. It has to be noted that the grating lobe is high in the beampattern transition band, but quite low $(<0.02)$ for

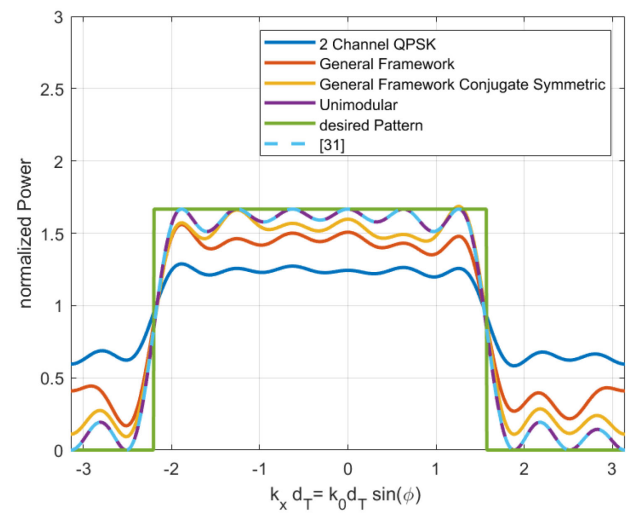

Fig. 4. Beampattern synthesis with Rectangular desired beampattern. The offset in the designed beampattern is typical for multiplexed signal designs.

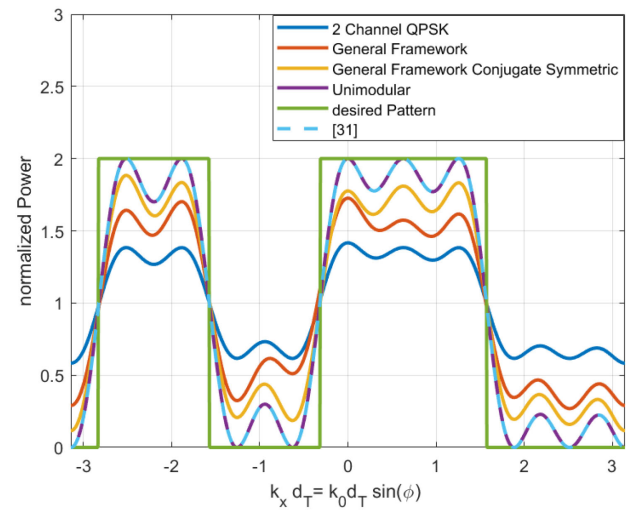

Fig. 5. Beampattern synthesis with two rectangular main lobes as desired pattern: difference in patterns is justified by the multiplexing design constraint.

the region of interest, i.e., target location within the beam. This normalized grating lobe demonstrates the effectiveness of $\mathrm{BCD}$ approach in terms of cross-correlation properties and virtual MIMO performance.

3) Design of Arbitrary Beam-Pattern: Fig. 4 and Fig. 5 illustrate the output of the algorithm for two different desired radiation patterns with different dictionaries. Common to both beampatterns is the offset (in amplitude) with respect to the desired pattern. This offset is justified by multiplexing, which leads inevitably to a non-identity dictionary matrix. The restriction to positive coefficients $\mathbf{v}$, leads, in turn, to a DC value in the radiation pattern design.

The comparison of our approach and [31] in Figs. 4 and 5 demonstrates that [31] result is equivalent to our unimodular signal design approach. The difference lies in the signal construction, where our approach takes into account non-zero columns in the signal matrix and further the beampattern is allowed to take on any shape without introducing an amplitude modulation. Therefore, our approach can be seen as a generalization of [31], where we take signal design constraints like general multiplexing and discrete phase modulation into account without limiting the beampattern shape. 


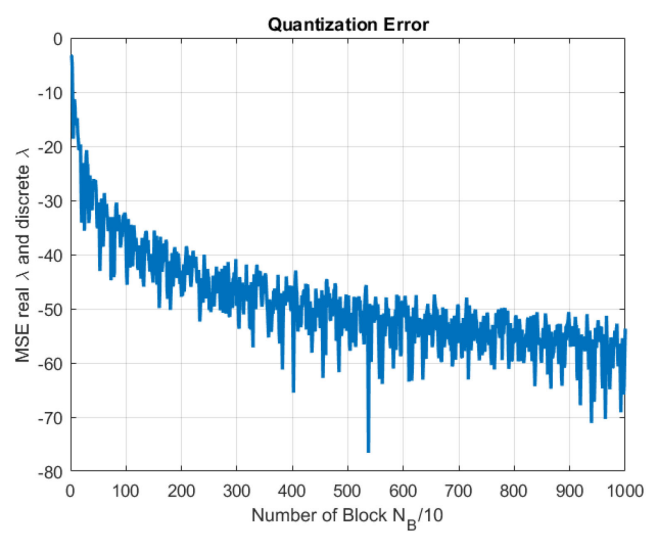

Fig. 6. Error between optimization output $\mathbf{v}$ and its discretized version as a function of number of blocks for the desired beampattern in Figure 4.

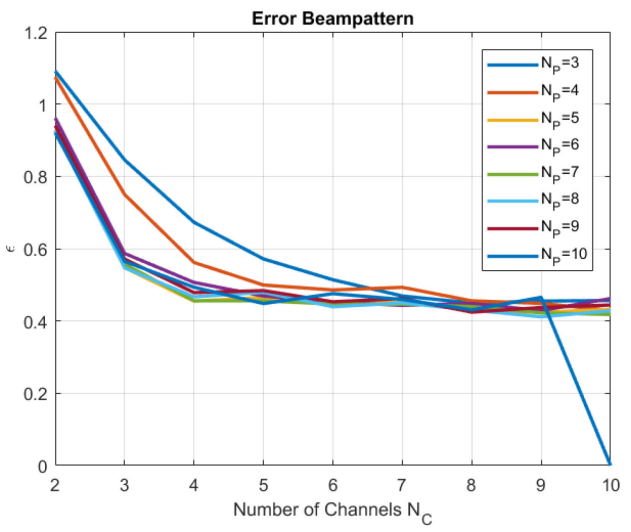

Fig. 7. Beampattern error as a function of number of phase stages $\left(N_{p}\right)$ and channels $\left(N_{c}\right)$ for the beampattern in Figure 4 . The higher the $N_{p}, N_{c}$ available in hardware, the better is the approximation of desired beampattern.

4) Impact of System Parameters on Beam-Pattern Design: Since the output of optimization are positive coefficients, discretization is necessary to satisfy the integer constraint. The rounding influences the beampattern output and Fig. 6 illustrates the effect on quantization. Higher the number of blocks, lower is the error in rounding the coefficients $\mathbf{v}$. Therefore, the ratio of nonzero elements of $\mathbf{v}$ and the number of blocks $N_{B}$ determines the fidelity of reproduction of each nonzero element of $\mathbf{v}$. Since the algorithm optimizes for sparsity in $\mathbf{v}$, the solution tends to have a fine resolution of $\mathbf{v}$. Further, enhanced sparsity in $\mathbf{v}$, in turn, affects the number of blocks which are needed for appropriate beampattern design. In derogation from the standard simulation setup, the number of phase stages and channels can be chosen arbitrary. Fig. 7 depicts the dependence of the radiation pattern error $\epsilon$ on $N_{P}$ and $N_{c}$ for a randomly initialized dictionary matrix, except for the unimodular case, where the dictionary matrix becomes identity. It can be seen that the error decreases if the number of channels and phase stages increase, leading to more degrees of freedom in the beampattern design. Further, the unimodular setup demonstrates that a proper choice of the dictionary matrix improves the algorithm output significantly. Fig. 8 illustrates that the improvement in beampattern design

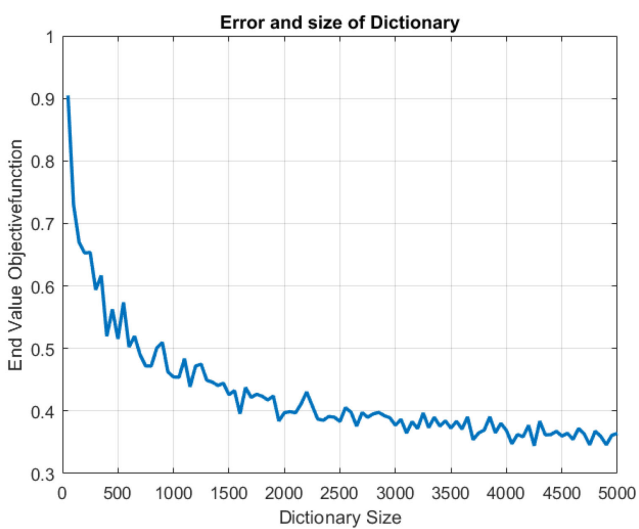

Fig. 8. Beampattern error as a function of dictionary size for the beampattern in Figure 4, $N_{P}=N_{c}=6$. The randomly initialized dictionary contains statistically more suitable atoms when its size is large.

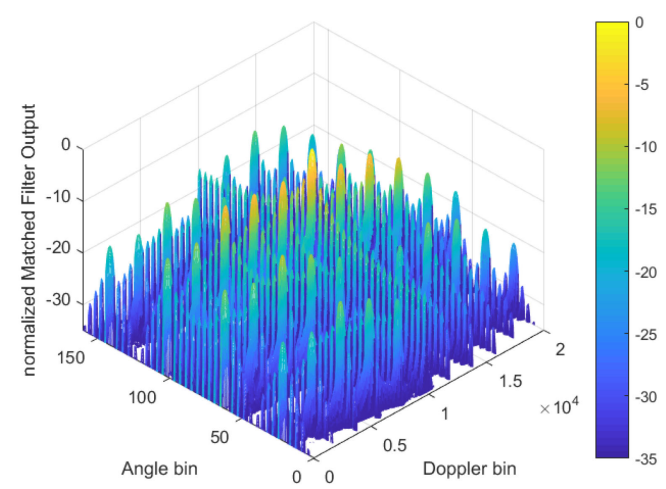

Fig. 9. Angle-Doppler ambiguity function for the block circulant signal matrix. Due to linear PCM, high sidelobes appear in the angle-Doppler plane.

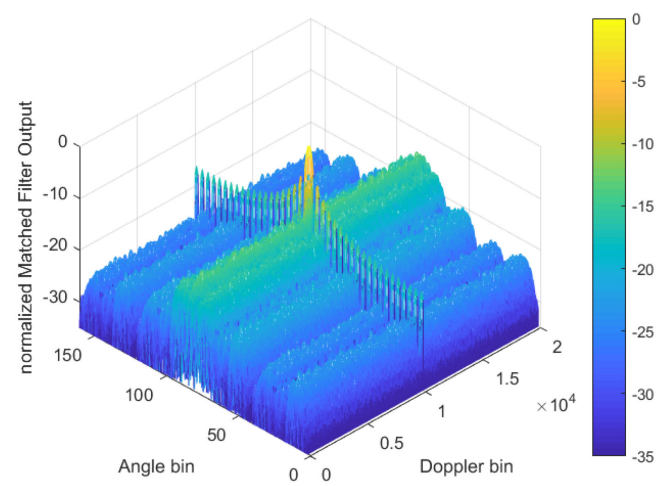

Fig. 10. Angle-Doppler ambiguity function for the randomized signal matrix. The sidelobes transform to a flat sidelobe floor.

when the size of the randomly initialized dictionary is increased for a fixed $N_{P}=N_{c}=6$. The probability of selecting good atoms increases with the dictionary size and the optimization provides better results.

5) Angle-Doppler Coupling and Mitigation: Fig. 9 shows the angle-Doppler ambiguity function for the proposed signal. As expected and discussed in relevant TDM-MIMO literature, angle-Doppler coupling appears. This can be seen from the 
number of peaks in the ambiguity function, which tend to restrict the unambiguous Doppler resolution. In contrast to Fig. 9, Fig. 10 demonstrates that even for the multiple antenna/ phase configuration, the randomized PCM procedure holds without disturbing the radiation pattern. It can be seen that the randomized angle-Doppler ambiguity function is similar to the TDM case, including the sinc characteristic due to the uniform power constraint. Since the transmitted signals are correlated, the ambiguity function changes in terms of sidelobes in accordance to the desired radiation pattern as described in (59). Further, the mitigation of the coupling is also evident from the figure.

\section{CONCLUSION}

The proposed beampattern design framework provides an architecture and methodology for designing inter-pulse modulated transmitted signals for MIMO systems with accent on cost-optimization and performance enhancement. The architecture generalizes the virtual TDM-MIMO architecture to offer beampattern shaping capability while maintaining enhanced target discrimination at the receiver; it also imposes discrete phase constraint on the modulation signals to enhance power efficiency. Further, a block circulant structure is imposed for the inter-pulse modulation signal to ease the optimization and enable it for adaptive designs. Leveraging on the signal structure and a dictionary based approach, the framework addresses the signal design through convex optimization. The crucial issue of angleDoppler coupling inherent in multiplexed signals is analyzed and a mitigation based on randomization is proposed. Centrality of dictionary matrix on the performance is depicted and dictionary design examples presented for special cases. The paper, thus provides a comprehensive investigation of the block circulant signal design for transmit beamforming in cost-optimized high performance MIMO radar, thus offering a solution to commercial applications including automotive.

\section{REFERENCES}

[1] A. Benjamin, "Imaging radar- the one sensor to rule them all," [Online]. Available: https://www.autonomoustechtlv.com/Portals/90/Amit.pdf

[2] D. Cerutti-Maori and I. Sikaneta, "A generalization of DPCA processing for multichannel SAR/GMTI radars," IEEE Trans. Geosci. Remote Sens., vol. 51, no. 1, pp. 560-572, Jan. 2013.

[3] J. R. Guerci, J. S. Bergin, R. J. Guerci, M. Khanin, and M. Rangaswamy, "A new MIMO clutter model for cognitive radar," in Proc. IEEE Radar Conf., 2016, pp 1-6.

[4] E. Fishler, A. Haimovich, R. Blum, and D. Chizihik, "MIMO radar: An idea whose time has come," in Proc. IEEE Radar Conf., Apr. 2004, pp. 71-78.

[5] F. Rusek et al., "Scaling up MIMO: Opportunities and challenges with very large arrays," IEEE Signal Process. Mag., vol. 30, no. 1, pp. 40-60, Jan. 2013

[6] D. Bliss, K. Forsythe, and G. Fawcett, "MIMO radar: Resolution and performance and and waveforms," in Proc. ASAP, 2006.

[7] F. Robey, S. Coutts, D. Weikle, J. McHarg, and K. Cuomo, "MIMO radar theory and experimental results," in Proc. 38th Asilomar Conf. Signals Syst. Comput., vol. 1, pp. 305-309, Nov. 2004.

[8] M. Soltanalian and P. Stoica, "Computational design of sequences with good correlation properties," IEEE Trans. Signal Process., vol. 60, no. 5, pp. 2180-2193, May 2012.

[9] P. Stoica, H. He, and J. Li, "New algorithms for designing unimodular sequences with good correlation properties," IEEE Trans. Signal Process., vol. 57, no. 4, pp. 1415-1425, Apr. 2009.

[10] M. Soltanalian and P. Stoica, "Designing unimodular codes via quadratic optimization," IEEE Trans. Signal Process., vol. 62, no. 5, pp. 1221-1234, Mar. 2014.
[11] J. Li, P. Stoica, and X. Zheng, "Signal synthesis and receiver design for MIMO radar imaging," IEEE Trans. Signal Process., vol. 56, no. 8, pp. 3959-3968, Aug. 2008.

[12] M. V. Kilian Rambach and B. Yang, "Optimal time division multiplexing schemes for DOA estimation of a moving target using a colocated MIMO radar," in Proc. IEEE Int. Symp. Signal Process. Inf. Technol., 2014, pp. $108-113$.

[13] G. Cui, H. Li, and M. Rangaswamy, "MIMO radar waveform design with constant modulus and similarity constraints," IEEE Trans. Signal Process., vol. 62, no. 52, pp. 343-353, Jun. 2014.

[14] O. Aldayel, V. Monga, and M. Rangaswamy, "Successive QCQP refinement for MIMO radar waveform design under practical constraints," IEEE Trans. Signal Process., vol. 64, no. 14, pp. 3760-3774, Jul. 2016.

[15] M. Soltanalian, H. Hu, and P. Stoica, "Single-stage transmit beamforming design for MIMO radar," Signal Process., vol. 102, pp. 132-138, 2014.

[16] K. Alhujaili, V. Monga, and M. Rangaswamy, "Quartic gradient descent for tractable radar slow-time ambiguity function shaping," IEEE Trans. Aerosp. Electron. Syst., vol. 56, no. 2, pp. 1474-1489, Apr. 2020.

[17] T. Aittomaki and V. Koivunen, "Low-complexity method for transmit beamforming in MIMO radars," in Proc. Int. Conf. Acoust. Speech, Signal Process., 2007, pp. II-305-II-308.

[18] C. Hammes, B. Shankar, Y. Nijsure, T. Spielmann, and B. Ottersten, "Random phase center motion technique for enhanced angle-Doppler discrimination using MIMO radars," in Proc. Eur. Signal Process. Conf., 2017, pp. 2221-2225.

[19] Y. Tong and A. Tennant, "A two-channel time modulated linear array with adaptive beamforming," IEEE Trans. Antennas Propag., vol. 60, no. 1, pp. 141-147, Jan. 2012.

[20] J. Guo, S. Qu, J. Hu, and Z. Nie, "A study on linear frequency modulation signal transmission by 4D antenna arrays," IEEE Trans. Antennas Propag., vol. 63, no. 12, pp. 5409-5416, Dec. 2015.

[21] J. Yang, W. Li, and X. Shi, "Phase modulation technique for fourdimensional arrays," IEEE Antennas Wireless Propag. Lett., vol. 13, pp. 1393-1396, Jul. 2014.

[22] P. Stoica, J. Li, and Y. Xie, "On probing signal design for MIMO radar," IEEE Trans. Signal Process., vol. 55, no. 8, pp. 4151-4161, Aug. 2007.

[23] C. Hammes, B. Shankar, and B. Ottersten, "Closed form discrete unimodular MIMO waveform design using block circulant decomposition," in Proc. IEEE Radar Conf., 2019, pp. 1-6.

[24] D. R. Fuhrmann and G. Antonio, "Transmit beamforming for MIMO radar systems using partial signal correlation," in Proc. 38th Asilomar Conf. Signals Syst. Comput., vol. 1, pp. 295-299, Nov. 2004.

[25] T. Aittomaki and V. Koivunen, "Signal covariance matrix optimization for transmit beamforming in MIMO radars," in Proc. Asilomar Conf. Signals Syst. Comput., 2007, pp. 182-186.

[26] D. Fuhrmann and G. S. Antonio, "Transmit beamforming for MIMO radar systems using signal cross-correlation," IEEE Trans. Aerosp. Electron. Syst., vol. 44, no. 1, pp. 171-186, Jan. 2008.

[27] S. Ahmed, J. S. Thompson, Y. R. Petillot, and B. Mulgrew, "Finite alphabet constant-envelope waveform design for MIMO radar," IEEE Trans. Signal Process., vol. 59, no. 11, pp. 5326-5337, Nov. 2012.

[28] S. Ahmed and M. Alouini, "MIMO radar waveform covariance matrix for high SINR and low side-lobe levels," IEEE Trans. Signal Process., vol. 62, no. 8, pp. 2056-2065, Apr. 2016.

[29] S. Sen, "PAPR-constrained pareto-optimal waveform design for OFDMSTAP radar," IEEE Trans. Geosci. Remote Sens., vol. 52, no. 6, pp. 3658-3669, Jun. 2014.

[30] G. Cui, X. Yu, V. Carotenuto, and L. Kong, "Space-time transmit code and receive filter design for colocated MIMO radar," IEEE Trans. Signal Process., vol. 65, no. 5, pp. 1116-1129, 2017.

[31] T. Bouchoucha, S. Ahmed, and M. Alouini, "DFT-based closed-form covariance matrix and direct waveform design for MIMO radar to achieve desired beampatterns," IEEE Trans. Signal Process., vol. 62, no. 9, pp. 2104-2113, Jan. 2017.

[32] J. Lipor, S. Ahmed, and M. Alouini, "Fourier-based transmit beampattern design using MIMO radar," IEEE Trans. Signal Process., vol. 62, no. 9, pp. 2226-2235, Apr. 2014.

[33] Z. Cheng, Z. He, S. Zhang, and J. Li, "Constant modulus waveform design for MIMO radar transmit beampattern," IEEE Trans. Signal Process., vol. 65, no. 18, pp. 4912-4923, Sep. 2017.

[34] T. Bouchoucha, S. Ahmed, T. Y. Naffouri, and M. Alouini, "Closed-form solution to directly design face waveforms for beampatterns using planar arrays," in Proc. IEEE Int. Conf. Acoust. Speech, Signal Process., pp. 2359-2363, 2015.

[35] X. Zhang, Z. He, L. Bacchus, and J. Yan, "MIMO radar transmit beampattern matching design," IEEE Trans. Signal Process., vol. 63, no. 8, pp. 2049-2056, Apr. 2015. 
[36] K. Alhujaili, V. Monga, and M. Rangaswamy, "Transmit MIMO radar beampattern design via optimization on the complex circle manifold," IEEE Trans. Signal Process., vol. 67, no. 13, pp. 3561-3575, 2019.

[37] C. Hammes, B. Shankar, and B. Ottersten, "Block circulant decomposition of cross-correlation matrix for transmit mimo beamforming," in Proc. 10th Sensor Array Multichannels Signal Process. Workshop, pp. 597-601, Jul. 2018.

[38] M. B. Kilani, Y. Nijsure, G. Gagnon, G. Kaddoum, and F. Gagnon, "Cognitive waveform and receiver selection mechanism for multistatic radar," IET Radar Sonar Navigation, vol. 10, no. 2, pp. 417-425, Feb. 2015.

[39] A. Aubry, A. DeMaio, A. Farina, and M. Wicks, "Knowledge-aided (potentially cognitive) transmit signal and receive filter design in signaldependent clutter," IEEE Trans. Aerosp. Electron. Syst., vol. 49, no. 1, pp. 93-117, Jan. 2013.

[40] R. Gray, Toeplitz and Circulant Matrices: A Review. Dept. Elect. Eng., Stanford University, [Online]. Available: https://ee.stanford.edu/ gray/toeplitz.pdf

[41] Y. Eldar and G. Kutyniok, Compressed Sensing: Theory and Applications. Cambridge, U.K.: Cambridge Univ. Press, 2012.

[42] S. Boyd and L. Vandenberghe, Convex Optimization. Cambridge, U.K.: Cambridge Univ. Press, 2004.

[43] P. R. Gill, A. Wang, and A. Molnar, "The in-crowd algorithm for fast basis pursuit denoising," IEEE Trans. Signal Process., vol. 59, no. 10, pp. 4595-4605, Oct. 2011.

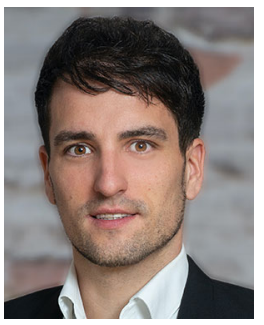

Christian Hammes (Member, IEEE) was born in Daun, Germany, in 1989. He received the M.Sc. degree in electrical engineering from the Trier University of Applied Science in 2015, and the Ph.D. degree in computer science from the University of Luxembourg in 2019. He worked on different topics with regards to $\mathrm{mm}$ Wave systems including Antenna designs, waveform design and sensor fusion of multiple decentral radar sensors together with other sensors like inertial measurement units and global positioning systems.

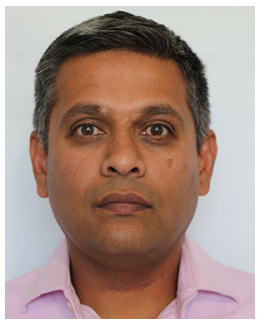

Bhavani Shankar M. R. (Senior Member, IEEE) received the master's and the Ph.D. degree in electrical communication engineering from the Indian Institute of Science, Bangalore in 2000 and 2007, respectively. He was a Postdoc with the ACCESS Linnaeus Centre, Signal Processing Lab, Royal Institute of Technology (KTH), Sweden from 2007 to September 2009. He joined SnT in October 2009 as a Research Associate and is currently a Research Scientist with SnT. He was with Beceem Communications, Bangalore from 2006 to 2007 as a Staff Design Engineer working on Physical Layer algorithms for WiMAX compliant chipsets. He was a Visiting Student with the Communication Theory Group, ETH Zurich, headed by Prof Helmut Blcskei during 2004. Prior to joining Ph.D., he worked on Audio Coding algorithms in Sasken Communications, Bangalore as a Design Engineer from 2000 to 2001. His research interests include design and optimization of MIMO communication systems, automotive radar and array processing, polynomial signal processing, satellite communication systems, resource allocation, game theory and fast algorithms for structured matrices. He is currently on the Executive Committee of the IEEE Benelux joint chapter on communications and vehicular technology, member of the EURASIP Technical Area Committee on Theoretical and Methodological Trends in Signal Processing and serves as Handling Editor for Elsevier Signal Processing. He was the co-recipient of the 2014 Distinguished Contributions to Satellite Communications Award from the Satellite and Space Communications Technical Committee of the IEEE Communications Society.

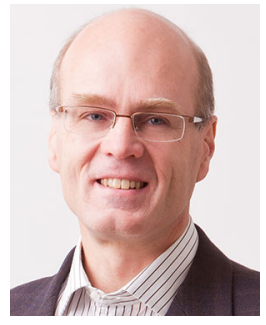

Björn Ottersten (Fellow, IEEE) was born in Stockholm, Sweden, in 1961. He received the M.S. degree in electrical engineering and applied physics from Linköping University, Linköping, Sweden, in 1986, and the Ph.D. degree in electrical engineering from Stanford University, Stanford, CA, USA, in 1990. He has held research positions with the Department of Electrical Engineering, Linköping University, the Information Systems Laboratory, Stanford University, the Katholieke Universiteit Leuven, Leuven, Belgium, and the University of Luxembourg, Luxembourg. From 1996 to 1997, he was the Director of Research with ArrayComm, Inc., a start-up in San Jose, CA, USA, based on his patented technology. In 1991, he was appointed Professor of signal processing with the Royal Institute of Technology (KTH), Stockholm, Sweden. From 1992 to 2004, he was the Head of the Department for Signals, Sensors, and Systems, KTH, and from 2004 to 2008 , he was the Dean of the School of Electrical Engineering, KTH. He is currently the Director for the Interdisciplinary Centre for Security, Reliability and Trust, University of Luxembourg. He was the recipient of the IEEE Signal Processing Society Technical Achievement Award in 2011 and the European Research Council advanced research grant twice, in 2009-2013 and in 2017-2022. He has coauthored journal papers that received the IEEE Signal Processing Society Best Paper Award in 1993, 2001, 2006, and 2013, and seven IEEE conference papers best paper awards. He has been a Board Member of IEEE Signal Processing Society, the Swedish Research Council and currently serves of the boards of EURASIP and the Swedish Foundation for Strategic Research. He has served was an Associate Editor for the IEEE Signal Processing Society and the Editorial Board of the IEEE Signal Processing Magazine. He is currently a member of the editorial boards of EURASIP Signal Processing Journal, EURASIP Journal of Advances Signal Processing and Foundations and Trends of Signal Processing. $\mathrm{He}$ is a fellow of EURASIP. 